\title{
Calculation of High Frequency Electromagnetic Field Coupling to Overhead Transmission Line above a Lossy Ground and Terminated with a Nonlinear Load
}

\author{
Jun Guo, Member, IEEE, Farhad Rachidi, Fellow, IEEE, Sergey Tkachenko, Senior Member, IEEE, \\ and Yan-zhao Xie, Member, IEEE
}

\begin{abstract}
High frequency electromagnetic fields such as those associated with EMP and IEMI can couple to overhead power lines. Since the height of the overhead power lines can be comparable or even larger than the smallest wavelength of typical EMP and IEMI pulses, the classical TL approximation might not be suitable for evaluating the line response. On the other hand, a traditional full wave solver (e.g. MoM) is computationally inefficient, especially when dealing with long lines. To address these problems, we propose an efficient method to handle the high frequency electromagnetic field coupling to overhead lines with nonlinear loads above a lossy ground. In the proposed method, the asymptotic approach is adopted and extended to the case of a lossy ground, which handles the problem in a semi-analytical way and has a much higher computational efficiency in the case of long lines. Although the proposed method applies in the frequency domain, the case of nonlinear loads can be considered through a combination with the time marching method. The proposed method is validated with several numerical examples.
\end{abstract}

Index Terms - High frequency electromagnetic field; overhead transmission line, transient response; asymptotic method; distributed parameter circuits,

\section{INTRODUCTION}

$\mathrm{O}$ VERHEAD transmission lines are one of the essential components in power systems. High frequency electromagnetic fields such as electromagnetic pulse (EMP) produced by a high-altitude nuclear burst (e.g., [1]- [2]) can induce significant overvoltage and currents, which may cause various effects such as short interruptions, voltage sags and even damage to power components, especially for distribution networks [3]. Furthermore, there is an increased concern about intentional electromagnetic interferences (IEMI) to power systems generated by high power transient electromagnetic

Jun Guo and Yan-zhao Xie are with the State Key Laboratory of Electrical Insulation and Power Equipment, National Center for International Research on Transient Electromagnetics and Applications, School of Electrical Engineering, Xi'an Jiaotong University, Xi'an 710049, China (e-mail: junguo@mail.xjtu.edu.cn; yzxie@mail.xjtu.edu.cn). sources such as compact radiation systems (e.g., [4]). Therefore, it is important to predict the response of overhead power lines.

The problem of high-frequency electromagnetic field coupling to transmission lines has been thoroughly studied in the past decades (see a review in [5]). One of the commonlyused methods is the classical transmission line (TL) theory [6][10]. This method is based on the transmission line approximation, where the line response is assumed to be the quasi transverse electromagnetic (TEM), and the high frequency effects (e.g. radiating modes or leaky modes) are neglected. The coupling equations resulting from the classical TL model [11]-[13] can be solved with a relatively low computational cost, and can provide the accurate solutions in the case when the line cross-sectional dimensions are electrically small, namely, smaller than about one tenth of the minimum wavelength of the incoming wave. This requirement might not be satisfied for the case of high frequency electromagnetic fields [14]. For example the IEC standard EMP waveform is characterized by a minimum significant wavelength of about $3 \mathrm{~m}$, which is smaller than the height of typical overhead power lines, which is about $10 \mathrm{~m}$ for distribution lines and even higher for the high voltage transmission lines.

To obtain an accurate solution in the case that the cross section of the line is comparable to the wavelength, the antenna theory is commonly used [15]. This method is based on the thinwire approximation, and can take into account the high frequency effects and provide an accurate solution for the high frequency field illumination. In general, a numerical full-wave technique, e.g., method of moment (MoM) should be applied to solve the mixed potential integral equation (MPIE), thus, leading to a high computational cost especially in the case of overhead power lines whose length extends typically to several kilometers.

Farhad Rachidi is with the Electromagnetic Compatibility Laboratory, Swiss Federal Institute of Technology, Lausanne, CH-1015, Switzerland (e-mail: farhad.rachidi@epfl.ch).

Sergey Tkachenko is with the Institute for Fundamental Electrical Engineering and EMC (Chair of Electromagnetic Compatibility now), Ottovon-Guericke University Magdeburg, Magdeburg, Germany (e-mail: Sergey.Tkachenko@ovgu.de). 
In order to improve the computational efficiency while keeping the high accuracy of the solutions when modeling the high frequency electromagnetic field coupling to overhead lines, some researchers have proposed the so called enhanced or fullwave TL method. Tkachenko et al. proposed a TL-like method to handle the high frequency electromagnetic field coupling to a finite length line with open circuit terminals above a perfectly conducting ground that is based on the perturbation theory [16], where the solutions can be obtained iteratively. Tkachenko et $a l$. then proposed an asymptotic method to obtain the response of a finite length line terminated by arbitrary linear loads above a PEC ground in a semi-analytical way [17]. The singularity expansion method (SEM) was also adopted to obtain the analytical solution in time domain [18], which can be applied to the case when the finite length line is terminated on open circuit or short circuit terminations. Poljak et al. proposed a numerical method to handle the case of a finite line above a lossy ground with open circuit terminals [14].

Since the power lines are typically terminated by nonlinear loads for the sake of protection, it is important to analyze the response behavior and the protection effects by modeling the transmission line with the nonlinear loads. Therefore, to enhance the applicability of the model to real scenarios, this paper aims to propose a method to solve the high frequency electromagnetic field coupling to overhead lines with nonlinear loads above a lossy ground. In the proposed method, the asymptotic approach, characterized by a high computational efficiency, is adopted and extended to the case of a lossy line. The general solution along the asymptotic region in the lossy ground case is developed. First, the scattering coefficients and the reflection coefficients are fitted and calculated in the frequency domain. Then, the response along the entire line can be calculated by these obtained coefficients, allowing therefore a higher computational efficiency than classical full-wave solvers. Since the asymptotic method is based on a frequency domain approach, the time marching method which is a mixed frequency domain and time domain method is adopted to handle nonlinear loads. The proposed method can therefore accurately solve the problem of high frequency uniform plane wave (e.g. EMP) coupling to long overhead lines terminated with nonlinear loads above a lossy ground. Additionally, by using the electric field representation method [19]-[21], the proposed method can also be extended to the case nonuniform wave excitations (e.g. compact radiation systems in IEMI).

The remainder of this paper is organized as follows. Section II describes the basic concept of the proposed approach. Section III presents the results associated with several case studies to validate the proposed approach. Finally, Section IV presents a summary and general conclusions.

\section{BASIC CONCEPT OF THE PROPOSED APPROACH}

The problem to be investigated is shown in Fig. 1. We consider an overhead lossless conductor ${ }^{1}$ of length $L$, radius $r$ and height of $h$ above a lossy ground, illuminated by an external

\footnotetext{
${ }^{1}$ The conductor losses are negligible in typical overhead power lines [F. Rachidi, C.A. Nucci, M. Ianoz, C. Mazzetti, "Influence of a lossy ground on
}

electromagnetic field. The dielectric constant, electric conductivity and permeability for the air are $\varepsilon_{0}, 0$ and $\mu_{0}$, respectively, and the ground is characterized by its electric parameters, namely $\varepsilon_{g}, \sigma_{g}$ and $\mu_{0}$. The incoming wave is a uniform plane wave which has a polarization angle $\alpha$, azimuth angle $\theta$ and an elevation angle $\psi$. The terminal loads are $Z_{1}$ in the left end and $Z_{2}$ in the right end. Both of the loads are considered as lumped components connected in the vertical risers at a small height $\Delta / 2$ above the ground.

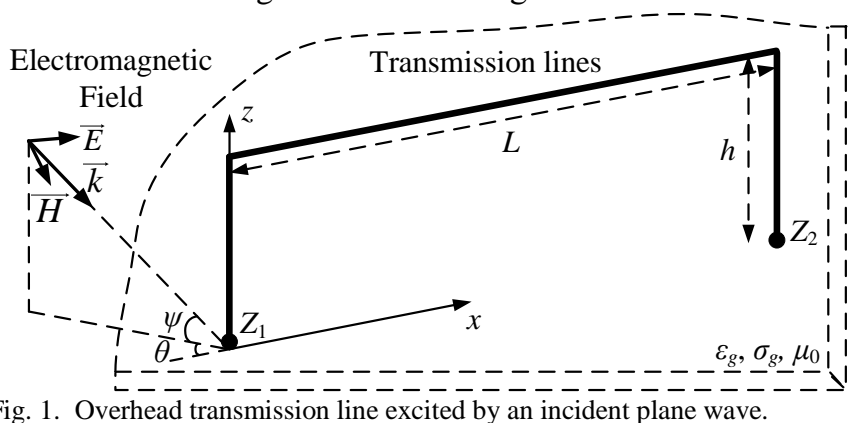

A. Mixed Potential Integral Equation (MPIE) for the Line

Since the conductor is considered as lossless, the $x$ component of the total electric field along the line is zero.

$$
E_{x}^{e}(x)+E_{x}^{s}(x)=0
$$

where $E_{x}{ }^{e}$ is the $x$-component of excitation electric field, and $E_{x}{ }^{s}$ is the $x$-component of scattered electric field. The expression for $E_{x}^{e}$ is

$$
\begin{aligned}
E_{x}^{e}(x, j \omega) & =E_{0}(j \omega)\left[\cos \alpha \sin \psi \cos \theta\left(e^{j k h \sin \psi}-R_{v} e^{-j k h \sin \psi}\right)\right. \\
& \left.+\sin \alpha \sin \theta\left(e^{j k h \sin \psi}+R_{h} e^{-j k h \sin \psi}\right)\right] e^{-j k \cos \psi \cos \theta} \\
& =E(j \omega) e^{-j k_{1} x}
\end{aligned}
$$

where $R_{v}$ and $R_{h}$ are the Fresnel ground reflection coefficients for vertical and horizontal polarizations, respectively. $k$ is the wave number in the free space that is $\omega / c$. The scattered electric field can be expressed as

$$
\vec{E}^{s}=-j \omega \vec{A}-\nabla \varphi
$$

where $A$ is the magnetic vector potential and $\varphi$ is the electric scalar potential. Expression (3) can be written for the $x$ component as

$$
E_{x}^{s}(x)=-j \omega A_{x}(x)-\frac{\partial \varphi(x)}{\partial x}
$$

where

$$
\begin{aligned}
& A_{x}(x)=\frac{\mu_{0}}{4 \pi} \int_{0}^{L} I\left(x^{\prime}\right) g\left(x, x^{\prime}\right) d x^{\prime} \\
& \varphi(x)=\frac{1}{4 \pi \varepsilon} \int_{0}^{L} q\left(x^{\prime}\right) g\left(x, x^{\prime}\right) d x^{\prime}
\end{aligned}
$$

where $q(x)$ is the charge distribution along the line, $I\left(x^{\prime}\right)$ denotes the induced current along the line, and $g(x, x)$ is the Green's function, whose expression can be found in [14].

The relationship of the charge distribution and the current can be written as

lightning-induced voltages on overhead lines", IEEE Trans. on Electromagnetic Compatibility, Vol. 38, No. 3, August 1996.]. 


$$
q=-\frac{1}{j \omega} \frac{d I}{d x}
$$

Inserting (7) into (6), we obtain

$$
\varphi(x)+\frac{1}{4 \pi \varepsilon j \omega} \int_{0}^{L} \frac{\partial I\left(x^{\prime}\right)}{\partial x^{\prime}} g\left(x, x^{\prime}\right) d x^{\prime}=0
$$

And inserting (1) and (5) into (4), we obtain

$$
\frac{\partial \varphi(x)}{\partial x}+j \omega \frac{\mu_{0}}{4 \pi} \int_{0}^{L} I\left(x^{\prime}\right) g\left(x, x^{\prime}\right) d x^{\prime}=E_{x}^{e}(x)
$$

Expressions (8) and (9) form the mixed potential integral equations that describe the induced response along the line. It is to be noted that expressions (8) and (9) are derived considering a finite line with open circuit terminals. For the case of arbitrary line terminations, the exact solution of the induced current can be determined by the solution of the complicated Pocklington's equation [10], [17]. Moreover, when the ground has a finite conductivity, the resulting equations would be much more complicated [14], [22]. To obtain the exact solutions using these equations, numerical full-wave methods (e.g., MoM) have to be applied, which are computationally inefficient.

To simplify the problem, the transmission line (TL) theory has been used. For a perfectly-conducting ground, and when the relationship between the line cross section and the incoming wave meets the TL assumptions, namely when $k h<\langle 1$ and $L>>h$, the following integral can be approximated as [ref to Tkachenko et al]

$$
\int_{0}^{h} I\left(x^{\prime}\right) g\left(x, x^{\prime}\right) d x^{\prime} \approx I(x) \int_{0}^{L} g\left(x, x^{\prime}\right) d x^{\prime} \approx 2 I(x) \ln \frac{2 h}{r}
$$

With the approximation of (10) and considering of the definitions of the electric scalar potential $\varphi$ and the scattered voltage $V^{s}$, equations (8) and (9) will reduce to

$$
\begin{gathered}
\frac{d I\left(x^{\prime}\right)}{d x^{\prime}}+\frac{4 \pi \varepsilon j \omega}{2 \ln \frac{2 h}{r}} V^{s}(x)=\frac{d I\left(x^{\prime}\right)}{d x^{\prime}}+j \omega C^{\prime} V^{s}(x)=0 \\
\frac{d V^{s}(x)}{d x}+\frac{j \omega \mu_{0} 2 \ln \frac{2 h}{r}}{4 \pi} I(x)=\frac{d V^{s}(x)}{d x}+j \omega L^{\prime} I(x)=E_{x}^{e}(x)
\end{gathered}
$$

Equations (11) and (12) are the field-to-transmission line coupling equation with the TL assumption in the Agrawal et al. form [12].

For a lossy ground case, equations (11) and (12) become

$$
\begin{aligned}
& \frac{d I\left(x^{\prime}\right)}{d x^{\prime}}+Y^{\prime} V^{s}(x)=0 \\
& \frac{d V^{s}(x)}{d x}+Z^{\prime} I(x)=E_{x}^{e}(x)
\end{aligned}
$$

where $Y^{\prime}$ and $Z^{\prime}$ are the per-unit-length (p.u.l.) transverse admittance and longitudinal impedance of the line [10], respectively.

$$
Y^{\prime}=\frac{j \omega C^{\prime} Y_{g}{ }^{\prime}}{j \omega C^{\prime}+Y_{g}{ }^{\prime}}, \quad Z^{\prime}=j \omega L^{\prime}+Z_{g}{ }^{\prime}
$$

The Sunde [23] approximation can be used to evaluate the ground impedance $Z_{g}{ }^{\prime}$

$$
\begin{aligned}
& Z_{g}{ }^{\prime} \approx \frac{j \omega \mu_{0}}{2 \pi} \ln \frac{1+\gamma_{g} h}{\gamma_{g} h}, \quad Y_{g}{ }^{\prime} \approx \frac{\gamma_{g}{ }^{2}}{Z_{g}{ }^{\prime}} \\
& \gamma_{g}=\sqrt{j \omega \mu_{0}\left(\sigma_{g}+j \omega \varepsilon_{g}\right)}
\end{aligned}
$$

\section{B. Asymptotic Method and Its Extension to the Case of a Lossy Ground}

When we consider a uniform plane wave coupling to an infinitely-long lossless conductor above a lossy ground, with the thin wire assumption (radius of the wire much smaller than the wavelength and the height of line), the current response along the line can be expressed analytically as [24]

$$
I_{\text {inf }}(x, j \omega)=\frac{E_{x}^{e}(j \omega)}{Z^{e}(j \omega)} e^{-j k_{1} x}=I_{0}(j \omega) e^{-j k_{1} x}
$$

where $Z^{e}$ and $I_{0}$ are the external impedance of the line and the current coefficient, respectively, which both depend on the line geometry and the frequency. Since $I_{0}$ is independent of the location $x$, the current response has an exponential distribution along the line.

When the line has a finite length, the problem can be solved iteratively and analytically using the perturbation theory [16]. To obtain the analytical formulation in time domain, the singularity expansion method can be applied [18]. This method adopts an iterative approach and is suitable for the more general case of terminals with arbitrary geometries and arbitrary linear loads. Moreover, to pursue a high computational efficiency, this problem can also be solved semi-analytically using the asymptotic method [17]. However, the above-mentioned methods are all based on the perfectly-conducting ground assumption.

In this section, the asymptotic method is extended to the case of a lossy ground. It can be seen from (17) that the current response is expressed as an exponential function of $x$ (along the infinite line). When the line is terminated on a load along a vertical riser, the solution becomes much more complicated, especially in the regions near the terminals. However, in the central part of the line, far enough from both ends, the highfrequency effects generated from the discontinuities would decrease very fast, so that this region can be treated asymptotically as an infinite line [17]. In this way, the entire line can be separated into three regions, as shown in Fig. 2. Region I is the region near the left terminal which contains the left terminal riser and a portion of the horizontal line, $0 \leq x<l_{b}-h$, Region III is the region in the right terminal which contains the right terminal riser and a portion of the horizontal line, $L$ $l_{b}+h<x \leq L$. Region II is the asymptotic region along the line, $l_{b^{-}}$ $h \leq x \leq L-l_{b}+h . l_{b}$ is total length of the terminal region which depends on the height $h$ and the frequency. Typically, a value of $l_{b}$ of about twice of the height can be adopted [17].

In the asymptotic region, the induced current can be expressed as

$$
I(x, j \omega)=I_{0}(j \omega) e^{-j k_{1} x}+F_{1}(x, j \omega)+F_{2}(x, j \omega)
$$

where $F_{1}$ and $F_{2}$, are unknown functions to be determined. 


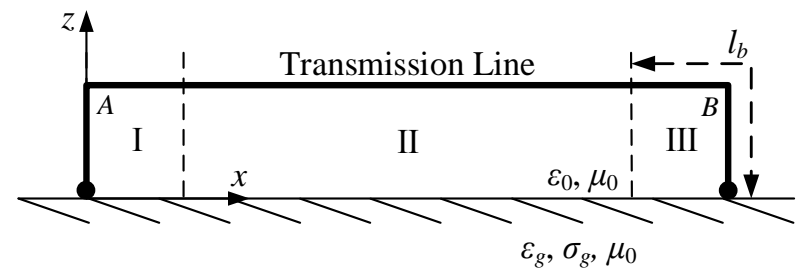

Fig. 2. The three regions along the transmission line (from [17]).

The first term in expression (18) corresponds to the response generated by the exciting field along an infinitely-long line that with the same geometry (height, radius and ground electrical parameters), and which can be obtained by using equation (17). The last two terms $F_{1}$ and $F_{2}$ in expression (18) represent forward and backward waves propagating from the terminal regions generated as a result of scattering and reflections. These two terms should have the same form as the solution for the same line excited by lumped voltage sources located at line ends. The solutions for the current along a conductor above a lossy ground have been presented in different forms (e.g., [25-29]). These methods involve the solution of the Sommerfeld integrals, which are computationally inefficient especially in the case of multiple-frequency simulation. Here we use a simplified approach which is described in what follows.

For the case of a perfectly-conducting ground, the current along the asymptotic region can be expressed as [17]

$$
I_{P}(x, j \omega)=I_{P, 0}(j \omega) e^{-j k \cos \psi x}+I_{P, 1}(j \omega) e^{-j k x}+I_{P, 2}(j \omega) e^{j k x}
$$

In order to take into account the presence of a lossy ground, we use the approximate approach in which the functions $F_{1}$ and $F_{2}$ are still expressed with exponential functions with $x$ as in the case of lossless ground, with the propagation constant evaluated by using the TL theory taking into account the lossy ground. In this way, the current solution along the asymptotic region can be expressed by

$$
I(x, j \omega)=I_{0}(j \omega) e^{-j k_{1} x}+I_{1}(j \omega) e^{-\gamma x}+I_{2}(j \omega) e^{\gamma x}
$$

where $\gamma$ is the line propagation constant is given by

$$
\gamma=\sqrt{Z^{\prime} Y^{\prime}}
$$

in which the p.u.l. parameter $Z^{\prime}$ and $Y^{\prime}$ are determined by using (15) and (16). Although these parameters and expressions are to be used within the TL theory, they can still provide accurate solutions for the current along the asymptotic region in a broad frequency range, as will be shown in Section III.

In order now to determine the expressions for $I_{0}, I_{1}$ and $I_{2}$, we follow the same approach adopted in [17], considering two semi-infinite lines. First, we consider a right semi-infinite length line, extending from $x=0$ to infinity, and having the same geometry as that in Fig. 2. When this line is illuminated by the exciting field, the induced current along the line can be expressed as

$$
I_{+}^{S}(x)=\left\{\begin{array}{cc}
I_{0} F_{+}^{S}(x) & 0 \leq x \leq l_{b}-h \\
I_{0} e^{-j k_{1} x}+S_{+} I_{0} e^{-\gamma x} & x>>l_{b}-h
\end{array}\right.
$$

where $S_{+}$is the scattering coefficient associated with the left terminal, $F_{+}{ }^{S}$ is the unknown function corresponding to the exact solution in the left terminal region.

Then, we consider that there is no exciting field and a hypothetical current $I_{2}$ ' flowing from right to left (homogeneous solution). The current along the line can be expressed as

$$
I_{+}^{R}(x)=\left\{\begin{array}{cc}
I_{2}{ }^{\prime} F_{+}^{R}(x) & 0 \leq x \leq l_{b}-h \\
R_{+} I_{2}{ }^{\prime} e^{-\gamma x}+I_{2}{ }^{\gamma} e^{\gamma x} & x>>l_{b}-h
\end{array}\right.
$$

where $R_{+}$is the reflection coefficient associated with the left terminal, $F_{+}{ }^{R}$ is an unknown function corresponding to the exact solution in the left terminal region.

The total solution of the right semi-infinite length line can be expressed as the sum of the above-mentioned two parts as

$$
I_{+}(x)=I_{+}^{S}(x)+I_{+}^{R}(x)
$$

Specifically, the total solution along the left terminal and the asymptotic region can be expressed as

$$
\begin{aligned}
& I_{\text {left }+}(x)=I_{0} F_{+}^{S}(x)+I_{2}{ }^{\prime} F_{+}^{R}(x) \\
& I_{\text {asy }+}(x)=I_{0} e^{-j k_{1} x}+\left(R_{+} I_{2}{ }^{\prime}+S_{+} I_{0}\right) e^{-\gamma x}+I_{2}{ }^{\prime} e^{\gamma x}
\end{aligned}
$$

In the same way, if we consider a left semi-infinite line along $-\infty<x \leq L$ above a lossy ground, the solution for the total current in the right terminal region and in the asymptotic region can be expressed as

$$
\begin{aligned}
& I_{\text {right }}(x)=I_{0} e^{-j k_{1} L} F_{-}^{S}(x-L)+I_{1}{ }^{\prime} F_{-}^{R}(x-L) \\
& I_{\text {asy- }}(x)=I_{0} e^{-j k_{1} x}+I_{1}^{\prime} e^{\gamma L} e^{-\gamma x}+\left(R_{-} I_{1} e^{-\gamma L}+S_{-} I_{0} e^{-j k_{1} L-\gamma L}\right) e^{\gamma x}
\end{aligned}
$$

where $I_{1}{ }^{\prime}$ is the hypothetical current flowing from left to right. $S$. and $R$ - are respectively the scattering and reflection coefficients associated with the right terminal, $F_{-}^{S}$ and $F_{-}^{R}$ are unknown functions corresponding to the exact solutions in the right terminal region. It is to be noted that the scattering and reflection coefficients are intrinsic coefficients of the line terminals which are independent on the length of line.

The expressions (25) and (26) are the solutions for the current associated with the two semi-infinite line. By imposing these two solutions along the asymptotic region to be equal, the expression for the unknown parameters $I_{1}$ and $I_{2}$ for a line length $L$ can be formulated as

$$
\begin{aligned}
& I_{1}(L)=I_{1}{ }^{\prime} e^{\gamma L}=\frac{S_{+}+S_{-} R_{+} e^{-j k_{1} L-\gamma L}}{1-R_{+} R_{-} e^{-2 \gamma L}} I_{0} \\
& I_{2}(L)=I_{2}{ }^{\prime}=\frac{S_{-} e^{-j k_{1} L-\gamma L}+S_{+} R_{-} e^{-2 \gamma L}}{1-R_{+} R_{-} e^{-2 \gamma L}} I_{0}
\end{aligned}
$$

From (27), it can be seen that the expression for the current along the asymptotic region can be obtained as a function of the scattering and reflection coefficients at the two line ends. To determine these coefficients, we follows the same approach as in [17] where two short auxiliary lines with the same geometry except for the length are simulated by a full-wave solver (e.g. MoM). In the simulation, the current response along the entire line is calculated. If we denote $L_{1}$ as the length of the auxiliary line 1 and $\Delta x$ the discretized space step along this auxiliary line, the current response along the asymptotic region can be expressed as

$$
I(n \Delta x)=I_{0} e^{-j k_{1} n \Delta x}+I_{1} e^{-\gamma n \Delta x}+I_{2} e^{\gamma n \Delta x}
$$

where 


$$
\begin{aligned}
& n=M+1, M+2, \ldots, N-M \\
& N=\frac{L_{1}}{\Delta x}, \quad M=\frac{l_{b}}{\Delta x}
\end{aligned}
$$

The coefficients $I_{0}, I_{1}$ and $I_{2}$ can be obtained easily from (29) by using a fitting method such as the least squares method [30]. Since the values of $I_{1}$ and $I_{2}$ depend on the line length, we denote the obtained coefficients as $I_{0}, I_{1}\left(L_{1}\right)$ and $I_{2}\left(L_{2}\right)$. Since there are four unknown coefficients, $S_{+}, S_{-}, R_{+}$and $R_{-}$, that need to be determined, another auxiliary line with a different length $L_{2}$ is also simulated, obtaining $I_{0}, I_{1}\left(L_{2}\right)$ and $I_{2}\left(L_{2}\right)$ using the same fitting way. By using the two groups of the fitted coefficients, the unknown scattering and reflection coefficients can be straightforwardly determined by solving (27). In addition, since the scattering and the reflection coefficients are independent of the line length, when determining these coefficients, the two auxiliary lines can be very short to ensure a high computational efficiency.

With the obtained coefficients, the expressions for the current at both terminal regions can also be formulated. In the case of the auxiliary line of length $L_{1}$, according to (25), the solution in the left terminal region is

$$
I_{\text {left }}^{L_{1}}(x)=I_{0} F_{+}^{S}(x)+I_{2}\left(L_{1}\right) F_{+}^{R}(x)
$$

In the case of the auxiliary line of length $L_{2}$, the solution in the left terminal region is

$$
I_{\text {left }}^{L_{2}}(x)=I_{0} F_{+}^{S}(x)+I_{2}\left(L_{2}\right) F_{+}^{R}(x)
$$

Since the current solution along the left terminal at these two cases are already obtained by using a full-wave approach, the unknown functions $F_{+}{ }^{S}$ and $F_{+}{ }^{R}$ can be easily determined using (30) and (31). Thus, the current response along the left terminal region of a line with any length $L$ can be calculated analytically as

$$
I_{\text {left }}(x)=\frac{I_{\text {left }}^{L_{2}}(x)-I_{\text {left }}^{L_{1}}(x)}{I_{2}\left(L_{2}\right)-I_{2}\left(L_{1}\right)}\left(I_{2}(L)-I_{2}\left(L_{2}\right)\right)+I_{\text {left }}^{L_{2}}(x)
$$

The expression for the current along the right terminal region can also be obtained in the same way.

$$
\begin{aligned}
& I_{\text {right }}(x)=\frac{I_{\text {right }}^{L_{2}}\left(x+L_{2}-L\right) e^{j k_{1}\left(L_{2}-L\right)}-I_{\text {right }}^{L_{1}}\left(x+L_{1}-L\right) e^{j k_{1}\left(L_{1}-L\right)}}{I_{1}\left(L_{2}\right) e^{j k_{1} L_{2}-\gamma L_{2}}-I_{1}\left(L_{1}\right) e^{j k_{1} L_{1}-\gamma L_{1}}} \\
& \times\left(I_{1}(L) e^{j k_{1} L-\gamma L}-I_{1}\left(L_{2}\right) e^{j k_{1} L_{2}-\gamma L_{2}}\right)+I_{\text {right }}^{L_{2}}\left(x+L_{2}-L\right) e^{j k_{1}\left(L_{2}-L\right)}
\end{aligned}
$$

Hence, the expressions of the current response along the entire line is obtained. By using these expressions, the current response of any line that has the same geometry but with any different length can be calculated analytically.

It is worth mentioning that the incoming wave was assumed to be a uniform plane wave where the exciting horizontal electric field along the line is an exponential function with $x$. It can be seen from (20) that the first term of the general solution along the asymptotic region corresponds to the solution of an infinitely-long line excited by the exciting field, which has the same form of exponential function as that of the horizontal exciting electric field along the line (2). Based on this characteristic, the proposed method can also be extended into the case that the incoming wave is a nonuniform wave, by fitting the horizontal electric field along the line with exponential functions using the electric field representation technique [19]-[21]. In doing so, the proposed method can be applied to the problem of IEMI excitation of overhead power lines. Additionally, this method can also be conveniently extended to the case of a multi-conductor transmission line [31]-[33].

\section{Asymptotic Method for the Case of a Lumped Voltage Source Excitation}

The problem of a line excited by a lumped voltage source located at an arbitrary location has been thoroughly discussed [34]-[36]. In this paper, we present a solution to solve this problem based on the asymptotic method.

Assume there are two lumped voltage sources $V_{1}$ and $V_{2}$ which are located at the two line ends. Since there is no exciting field, the general solution along the asymptotic region becomes

$$
I(x, j \omega)=I_{1}(j \omega) e^{-\gamma x}+I_{2}(j \omega) e^{\gamma x}
$$

Similar to the case when the exciting source is an incoming wave, two semi-infinite lines are considered. The solution along the right semi-infinite line excited by a lumped source located at the left end can be expressed as

$$
I_{+}^{S}(x)=\left\{\begin{array}{lc}
F_{+}^{P}(x) & 0 \leq x \leq l_{b}-h \\
P_{+} e^{-\gamma x} & x>>l_{b}-h
\end{array}\right.
$$

where $P_{+}$is a coefficient that describes the current solution along the asymptotic region as a result of a lumped source excitation at the left terminal. $F_{+}{ }^{P}$ is the unknown function corresponding to the exact solution in the left terminal region excited by a lumped voltage source.

Assuming there is no lumped voltage source at the left end and a hypothetical current $I_{2}{ }^{\prime}$ propagating from right to left (homogeneous solution), the current can be expressed as

$$
I_{+}^{R}(x)=\left\{\begin{array}{cc}
I_{2}{ }^{\prime} F_{+}^{R}(x) & 0 \leq x \leq l_{b}-h \\
R_{+} I_{2}{ }^{\prime} e^{-\gamma x}+I_{2}{ }^{\prime} e^{\gamma x} & x>>l_{b}-h
\end{array}\right.
$$

The total solution for the right semi-infinite line can be obtained as the sum of the above-mentioned two parts as

$$
\begin{aligned}
& I_{\text {left }+}(x)=F_{+}^{P}(x)+I_{2}{ }^{\prime} F_{+}^{R}(x) \\
& I_{\text {asy }+}(x)=\left(R_{+} I_{2}{ }^{\prime}+P_{+}\right) e^{-\gamma x}+I_{2}{ }^{\prime} e^{\gamma x}
\end{aligned}
$$

In the same way, when a left semi-infinite line is considered, the total current solution along the line can be expressed as

$$
\begin{aligned}
& I_{\text {right- }}(x)=F_{-}^{P}(x-L)+I_{1}{ }^{\prime} F_{-}^{R}(x-L) \\
& I_{\text {asy }-}(x)=I_{1}{ }^{\prime} e^{\gamma L} e^{-\gamma x}+\left(R_{-} I_{1}{ }^{\prime} e^{-\gamma L}+P_{-} e^{-\gamma L}\right) e^{\gamma x}
\end{aligned}
$$

where $P_{\text {- }}$ is the coefficient that describes the current solution along the asymptotic region as a result of a lumped source excitation at the right terminal, and $F_{-}{ }^{P}$ is the unknown function corresponding to the exact solution in the right terminal region, excited by a lumped voltage source at the right terminal.

By imposing these two solutions along the asymptotic region to be equal, the expressions for the unknown parameters $I_{1}$ and $I_{2}$ for line of length $L$ can be formulated as

$$
I_{1}(L)=\frac{P_{+}+P_{-} R_{+} e^{-\gamma L}}{1-R_{+} R_{-} e^{-2 \gamma L}}, \quad I_{2}(L)=\frac{P_{-} e^{-\gamma L}+P_{+} R_{-} e^{-2 \gamma L}}{1-R_{+} R_{-} e^{-2 \gamma L}}
$$

A similar procedure as in the case of a field excitation can be adopted. Two short auxiliary lines of length $L_{1}$ and $L_{2}$ excited 
by the two lumped sources are simulated by a full-wave solver. The coefficients $I_{1}\left(L_{1}\right), I_{1}\left(L_{2}\right), I_{2}\left(L_{1}\right)$ and $I_{2}\left(L_{2}\right)$ can be obtained by the least squares method. And then the coefficients $P_{+}, P_{-}$, $R_{+}$and $R_{-}$can be calculated by using (39). The expressions for the current in the two terminal regions can be formulated as

$$
\begin{aligned}
& I_{\text {left }}(x)=\frac{I_{\text {left }}^{L_{2}}(x)-I_{\text {left }}^{L_{1}}(x)}{I_{2}\left(L_{2}\right)-I_{2}\left(L_{1}\right)}\left(I_{2}(L)-I_{2}\left(L_{2}\right)\right)+I_{\text {left }}^{L_{2}}(x) \\
& I_{\text {right }}(x)=\frac{I_{\text {right }}^{L_{2}}\left(x+L_{2}-L\right)-I_{\text {right }}^{L_{1}}\left(x+L_{1}-L\right)}{I_{1}\left(L_{2}\right) e^{-\gamma L_{2}}-I_{1}\left(L_{1}\right) e^{-\gamma L_{1}}} \\
& \times\left(I_{1}(L) e^{-\gamma L}-I_{1}\left(L_{2}\right) e^{-\gamma L_{2}}\right)+I_{\text {right }}^{L_{2}}\left(x+L_{2}-L\right)
\end{aligned}
$$

Specifically, if there is only one lumped voltage source, the current solution can also be determined in a similar way. Assuming that there is a lumped voltage source $V$ located at the right end, the coefficients $F_{+}{ }^{P}$ and $P_{+}$would both reduce to zero. Since the other coefficients $R_{+}, R_{\text {- }}$ and $P_{\text {- }}$ are all independent of the lumped source at the left end, they can be calculated assuming that the line is excited by two identical lumped voltage sources $V$ located at the both ends of the line. The expressions of the coefficients $I_{1}$ and $I_{2}$ will be in this case

$$
I_{1}(L)=\frac{P_{-} R_{+} e^{-\gamma L}}{1-R_{+} R_{-} e^{-2 \gamma L}}, \quad I_{2}(L)=\frac{P_{-} e^{-\gamma L}}{1-R_{+} R_{-} e^{-2 \gamma L}}
$$

And the current in the terminal regions can be expressed as

$$
\begin{aligned}
& I_{\text {left }}(x)=\frac{I_{\text {left }}^{L_{2}}(x)-I_{\text {left }}^{L_{1}}(x)}{I_{2}\left(L_{2}\right)-I_{2}\left(L_{1}\right)} I_{2}(L) \\
& I_{\text {right }}(x)=\frac{I_{\text {right }}^{L_{2}}\left(x+L_{2}-L\right)-I_{\text {right }}^{L_{1}}\left(x+L_{1}-L\right)}{I_{1}\left(L_{2}\right) e^{-\gamma L_{2}}-I_{1}\left(L_{1}\right) e^{-\gamma L_{1}}} \\
& \times\left(I_{1}(L) e^{-\gamma L}-I_{1}\left(L_{2}\right) e^{-\gamma L_{2}}\right)+I_{\text {right }}^{L_{2}}\left(x+L_{2}-L\right)
\end{aligned}
$$

\section{Nonlinear Load}

The above-mentioned procedure is based on a frequencydomain approach and, therefore, cannot be directly applied to the case involving nonlinear loads. Methods allowing to take into account nonlinear loads within a frequency domain analysis have been discussed in the literature (e.g., [37]-[38]). This paper adopts a mixed frequency-time domain method that can be integrated into the proposed asymptotic method to solve the problem of a long overhead line terminated with a nonlinear load in an efficient manner. Assume that there is a lumped nonlinear load between two points $P_{a}$ and $P_{b}$ along the vertical riser of a terminal, as illustrated in Fig. 3(a). In this case, the Norton equivalent circuit between the two points $P_{a}$ and $P_{b}$ can be determined (see Fig. 3(b)), in which $I_{s c}$ is the equivalent Norton source which is equal to the current at the same point for a short-circuit termination $(Z=0)$, and $Y_{\text {in }}$ is the equivalent admittance of the circuit.

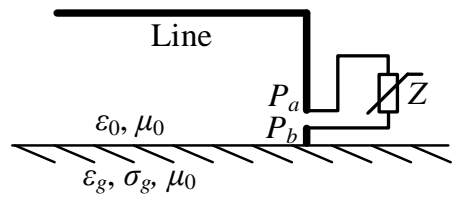

(a) Configuration

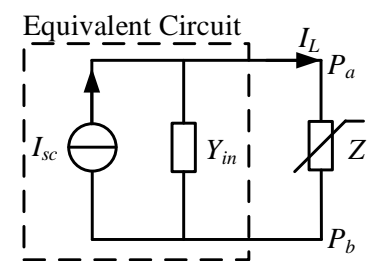

(b) Norton equivalent circuit
Fig. 3. The (a) configuration and the (b) Norton equivalent circuit of the nonlinear load terminated.

The relationship of the current in Fig. 3(b) can be simply expressed as

$$
I_{s c}(j \omega)=I_{L}(j \omega)+Y_{i n}(j \omega) U_{L}(j \omega)
$$

The relationship between the voltage and current on the load in time domain reads

$$
u_{L}(t)=F\left[i_{L}(t)\right]
$$

where $F$ is the $U-I$ characteristic of the nonlinear load.

The time domain formulation of (43) can be expressed as

$$
\begin{aligned}
& i_{s c}(t)=i_{L}(t)+y_{i n}(t) * F\left[i_{L}(t)\right] \\
& =i_{L}(t)+\int_{-\infty}^{t} y_{\text {in }}\left(t-t^{\prime}\right) F\left[i_{L}\left(t^{\prime}\right)\right] d t^{\prime}
\end{aligned}
$$

Equation (45) can be re-arranged into the following form, in which $\Delta t$ is chosen to be sufficiently small

$$
\begin{aligned}
& i_{s c}(t)=i_{L}(t)+\int_{-\infty}^{t-\Delta t} y_{i n}\left(t-t^{\prime}\right) F\left[i_{L}\left(t^{\prime}\right)\right] d t^{\prime}+\int_{t-\Delta t}^{t} y_{i n}\left(t-t^{\prime}\right) F\left[i_{L}\left(t^{\prime}\right)\right] d t^{\prime} \\
& \approx i_{L}(t)+\int_{-\infty}^{t-\Delta t} y_{i n}\left(t-t^{\prime}\right) F\left[i_{L}\left(t^{\prime}\right)\right] d t^{\prime}+y_{i n}(0) F\left[i_{L}(t)\right]
\end{aligned}
$$

Since the starting time of the exciting field is $t=0,(46)$ can be written as

$$
i_{L}(t)+y_{i n}(0) F\left[i_{L}(t)\right]=i_{s c}(t)-\int_{0}^{t-\Delta t} y_{i n}\left(t-t^{\prime}\right) F\left[i_{L}\left(t^{\prime}\right)\right] d t^{\prime}
$$

It can be seen from (47) that if the $U-I$ characteristic of the nonlinear load is determined, the current solution $i_{L}$ in time domain can be solved step by step using the time marching method.

To solve the current response of the line which is terminated by the nonlinear load in time domain, the key step is to determine the Norton equivalent circuit parameters, namely $i_{s c}$ and $y_{i n}$. In this paper, these two parameters are solved by the proposed asymptotic method in the frequency domain, and then transformed into time domain by using the IFFT method.

\section{NumERICAL VALIDATION OF THE PROPOSED ALGORITHM}

In this section, four numerical examples are considered to assess the proposed algorithm.

\section{A. Example 1: General Solution for the Current along the Asymptotic Region for the Case of a Lossy Ground}

In the first example, the assumption that the general solution of the current response along the asymptotic region in the lossy ground case could be approximately expressed by the exponential functions, and the propagation constant $\gamma$ could be evaluated by the TL theory is assessed. To this aim, we consider three cases which include both lumped and external field excitations. Since this example only aims at validating the general solution, the current along the horizontal part of the line $(x=0 \sim L)$ is entirely calculated by the general solution.

In the first case, a 100-m long, 10-m high overhead conductor of $1 \mathrm{~mm}$ diameter above a lossy ground is considered. The conductivity and the relative dielectric constant of the ground are assumed to be $0.01 \mathrm{~S} / \mathrm{m}$ and 10 , respectively. The terminal loads at both ends are $50 \Omega$. The line is excited by two $1-\mathrm{V}$ lumped voltage sources located at both terminals along the 
horizontal line (points $A$ and $B$ in Fig. 2). The frequency of the lumped voltage sources is $200 \mathrm{MHz}$. It should be noted that in this case the wavelength is $1.5 \mathrm{~m}$ which is much smaller than the height of the line, thus the classical TL approximation is not valid for this case. The current response along the line is simulated by the Numerical Electromagnetics Code (NEC-4) [39] which is a full-wave solver based on the MoM. Since there is no exciting field, the general solution along the asymptotic region is assumed given by (34). The current solutions along the whole asymptotic region is evaluated and then the unknown coefficients $I_{1}$ and $I_{2}$ are fitted by using the least squares method. The bound of the asymptotic region $l_{b}$ is set to $20 \mathrm{~m}$. When the unknown coefficients $I_{1}$ and $I_{2}$ are obtained, the current solution along the line are reconstructed using these fitted coefficients. The comparison between the original solutions obtained using NEC-4 and the reconstructed solutions using the fitted coefficients are shown in Fig. 4. The fitted coefficients are shown in Table I. It can be seen that in the asymptotic region the reconstructed waveforms agrees well with the original NEC-4 solutions.

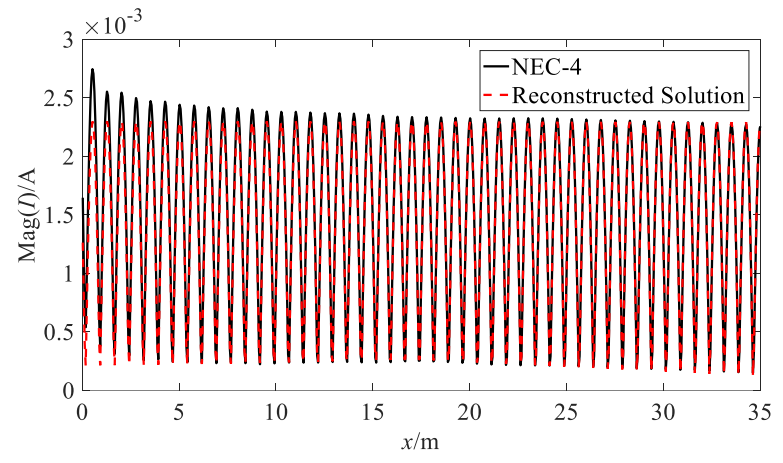

(a) $x=0 \sim 35 \mathrm{~m}$

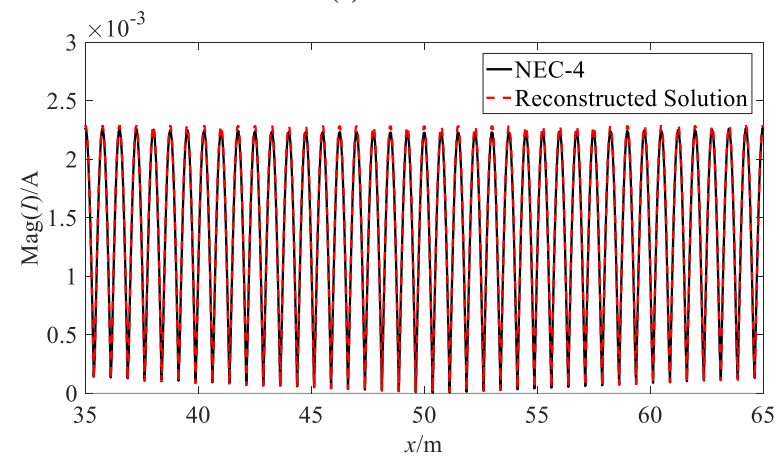

(b) $x=35 \sim 65 \mathrm{~m}$.

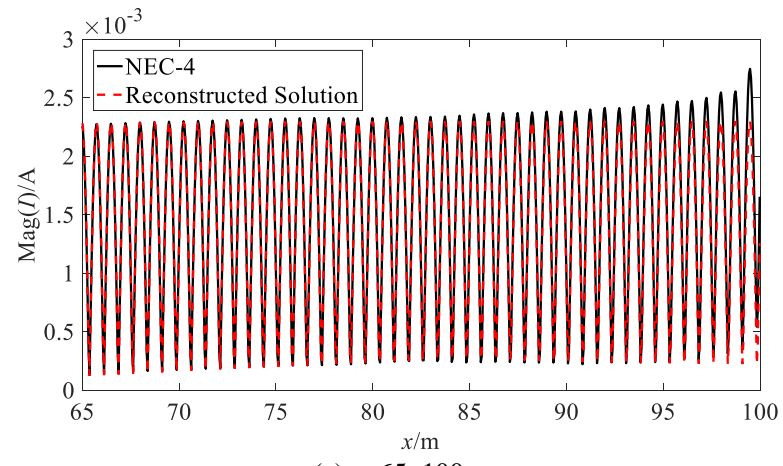

(c) $x=65 \sim 100 \mathrm{~m}$.

Fig. 4. Comparison between the original solutions obtained using NEC-4 and the reconstructed solution. The line is formed by a 100-m long, 10-m high, 1- $\mathrm{mm}$ diameter conductor above a ground of conductivity $0.01 \mathrm{~S} / \mathrm{m}$. Lumped source excitation with a frequency $f=200 \mathrm{MHz}$.

In the second case, the length of the line is $40 \mathrm{~m}$, the conductivity of the ground is $10^{-5} \mathrm{~S} / \mathrm{m}$ and the frequency of the lumped voltage sources is $500 \mathrm{MHz}$, while other parameters are the same as those in the first case. This case aims to validate the general solution in a more 'difficult' case characterized by a very poor ground conductivity and for a higher frequency. Note that such a low conductivity ground is not typical and is only used for the numerical validation of the proposed method.

In the same way, the current solution was calculated first using NEC-4, and the unknown coefficients were fitted by the least squares method, and then the current solution along the line was reconstructed by the obtained fitted coefficients. The comparison between the original NEC-4 solution and the reconstructed one are shown in Fig. 5. It can be seen that the reconstructed waveforms agree well with the NEC-4 solution in the asymptotic region.

TABLE I

THE FITTED COEFFICIENTS

\begin{tabular}{cc}
\hline$I_{1}$ & $I_{2}$ \\
\hline $11.65-4.21 \mathrm{i}$ & $1.47+10.52 \mathrm{i}$ \\
\hline
\end{tabular}

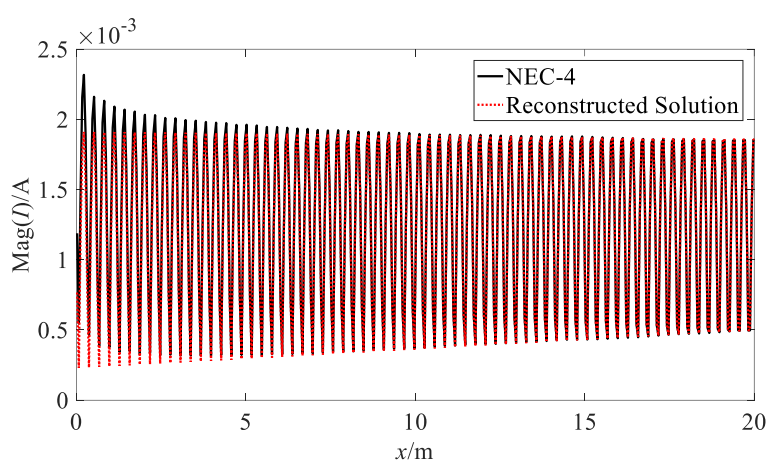

(a) $x=0 \sim 20 \mathrm{~m}$.

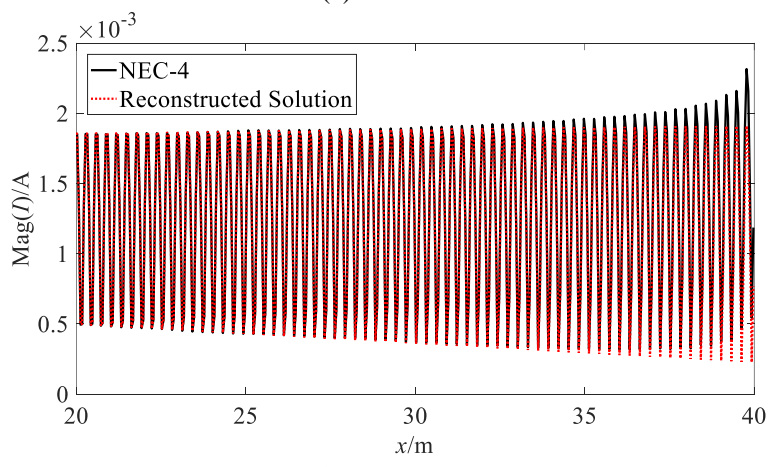

(b) $x=20 \sim 40 \mathrm{~m}$

Fig. 5. Comparison between the original solutions obtained using NEC-4 and the reconstructed solution using the fitted coefficients. The line is formed by a 40-m long, 10-m high, 1-mm diameter conductor above a ground of conductivity $10^{-5} \mathrm{~S} / \mathrm{m}$. Lumped source excitation with a frequency $f=500 \mathrm{MHz}$.

In the third case, the line is excited by an impinging field. The parameters of the line are the same as those in the second case, while the excitation is a plane wave with the amplitude of $1 \mathrm{~V} / \mathrm{m}$; the polarization angle, the azimuth angle and the elevation angle are $0^{\circ}, 0^{\circ}$ and $45^{\circ}$, respectively. The current response is calculated using both NEC-4 and the classical TL method. Then the reconstructed solution is also calculated by the fitted 
coefficients obtained from the solutions of NEC-4 using (29). The comparison between the induced currents calculated by NEC-4, classical TL method, and the reconstructed solution are shown in Fig. 6. It can be seen that the reconstructed solution agrees well with the solution obtained from the NEC-4 in the asymptotic region, whereas the solution obtained from the classical TL method is inaccurate.

These cases prove that the general solution in the asymptotic region expressed by (20) is an excellent approximation in the case of lumped and external field excitations. Moreover, the propagation constant estimated using the TL theory could provide the solutions with a high accuracy along the asymptotic region. The proposed approach is even reasonable for a 'difficult' case characterized by an unusually poor ground conductivity and for a broad frequency range covering a spectrum which extends to frequencies much beyond those of a typical EMP or even some of the IEMI sources. Above all, this example shows that the proposed approach is sufficiently reasonable for the typical case of EMP coupling to a distribution power line above a lossy ground.

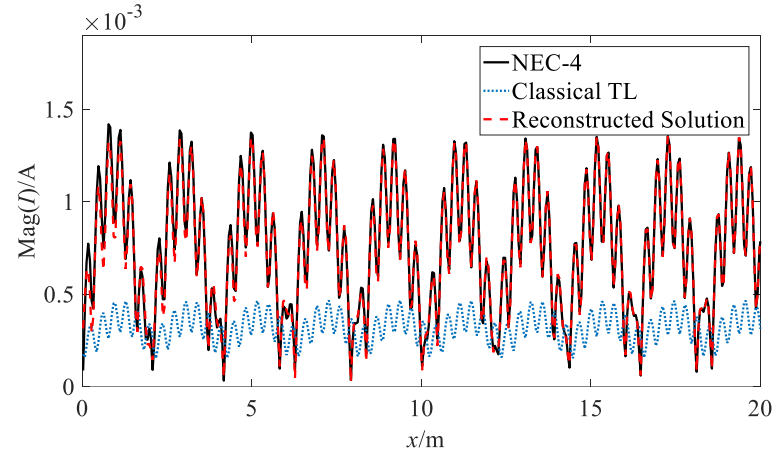

(a) $x=0 \sim 20 \mathrm{~m}$.

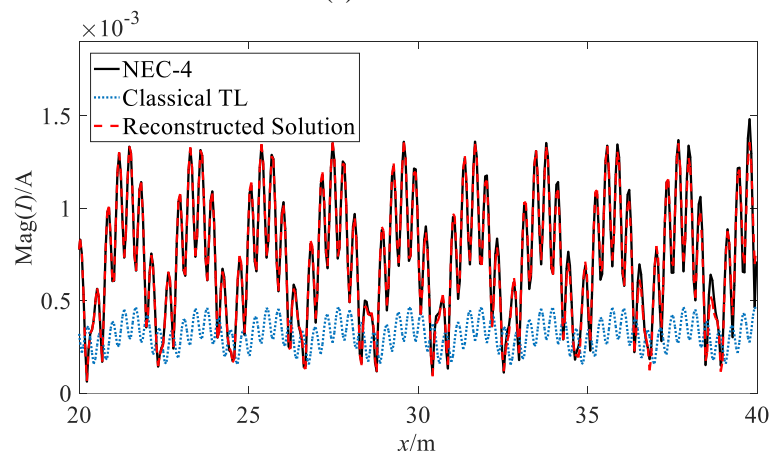

(b) $x=20 \sim 40 \mathrm{~m}$.

Fig. 6. Comparison between the original solutions obtained using NEC-4, the solution obtained using the classical TL theory and the reconstructed solution using the fitted coefficients. The line is formed by a 40-m long, 10-m high, 1$\mathrm{mm}$ diameter conductor above a ground of conductivity $10^{-5} \mathrm{~S} / \mathrm{m}$. The line is excited by a $1-\mathrm{V} / \mathrm{m}, 500-\mathrm{MHz}$ plane wave. The polarization angle, the azimuth angle and the elevation angle are $0^{\circ}, 0^{\circ}$ and $45^{\circ}$, respectively.

\section{B. Example 2: Long Overhead Line above a Lossy Ground Illuminated by an Exciting Field}

In the second example, the proposed method is entirely validated for several cases. Therefore, the current in the terminal regions are also taken into account.

In the first case, we consider a 200-m long, 10-m high, 1-mm diameter conductor above a lossy ground. The ground conductivity and relative permittivity are $0.01 \mathrm{~S} / \mathrm{m}$ and 10 , respectively. The terminal loads at both ends are $50 \Omega$. The line is excited by a plane wave with an amplitude of $1 \mathrm{~V}$; the polarization angle, the azimuth angle and the elevation angle are $0^{\circ}, 0^{\circ}$ and $45^{\circ}$, respectively. Since both vertical risers are taken into account, we use coordinate $l$ that describes the location along the line including both risers. In this way, the coordinates of the load $Z_{1}$ which is at $x=0, y=\Delta / 2$ corresponds to $l=\Delta / 2$, and that of the load $Z_{2}$ which is at $x=L, y=\Delta / 2$ corresponds to $l=L+2 h-\Delta / 2$. The line response was evaluated using NEC-4 and the proposed method. The current responses along the entire line at the frequency of $100 \mathrm{MHz}$ are shown in Fig. 7. It can be seen that the solution along the entire line calculated using the proposed approach agrees remarkably well with that the results obtained using NEC-4. The lengths of the two auxiliary lines used in the example were $60 \mathrm{~m}$ and $61.5 \mathrm{~m}$, respectively. Figures 8-11 present similar comparisons but considering longer lines ( $400 \mathrm{~m}$ and $1000 \mathrm{~m}$ ). It can be seen that the proposed method allows to reproduce with a reasonable accuracy the induced currents along the considered longer lines.

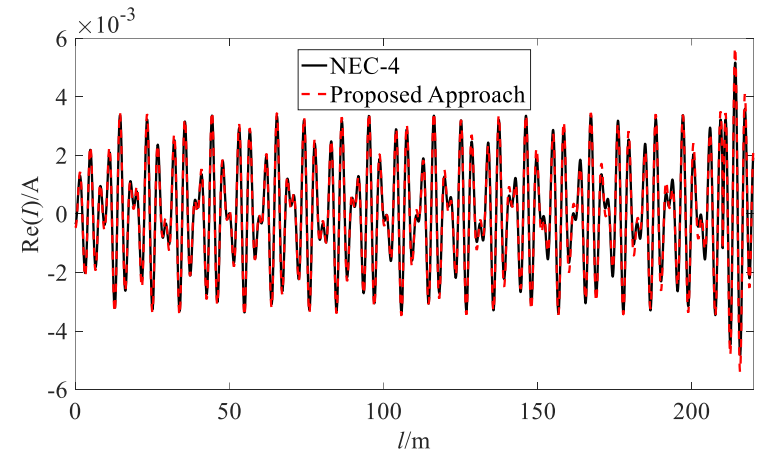

(a) Real part of the solution.

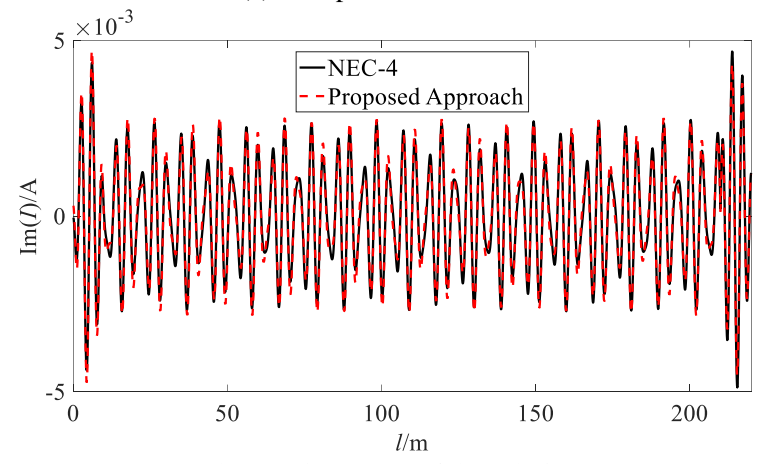

(b) Imaginary part of the solution.

Fig. 7. Comparison between the proposed approach and results obtained using NEC-4. Induced current on a 200-m long, 10-m high, 1-mm diameter conductor above a ground of conductivity $10^{-2} \mathrm{~S} / \mathrm{m}$ and relative permittivity 10 . The line is excited by a $1-\mathrm{V} / \mathrm{m}, 100-\mathrm{MHz}$ plane wave. The polarization angle, the azimuth angle and the elevation angle are $0^{\circ}, 0^{\circ}$ and $45^{\circ}$, respectively.

To quantify further the validity range of the proposed method as a function of the frequency, the relative error for the induced current at the line end with respect to the NEC results are evaluated using the following expression

$$
\varepsilon=\frac{|| I_{n}|-| I_{a}||}{\max \left(|I|_{n},\left|I_{a}\right|\right)}
$$

where $I_{n}$ is the magnitude of the current result obtained from NEC-4, and $I_{a}$ is the magnitude of the current result obtained from the proposed method. 
The relationship between the relative error and the frequency is shown in Fig. 12 for the case of the 1000-m long line. It can be seen from the figure that the relative error increases with the frequency. However, the results show that the relative error remains relatively low up to a frequency of about $250 \mathrm{MHz}$, which means that the proposed method is suitable for the simulation of HEMP coupling to overhead lines since significant frequency components of HEMP are within 100 $\mathrm{MHz}$ or so. To increase the accuracy for higher frequencies (e.g. $>300 \mathrm{MHz})$, other more accurate formulas for the ground impedance (e.g., [40]) can be used. This issue requires more indepth investigations and will be the subject of future work.

The computation time of the proposed approach for the three considered line lengths are reported in Table II. In the same table, the same figures related to NEC-4 are also reported for comparison. In the calculation, the discretization step along the line was $0.1 \mathrm{~m}$. The adopted software were NEC-4 and Matlab, which run on a PC with $3.2 \mathrm{GHz}$ CPU and $16 \mathrm{~GB}$ RAM. It can be seen that the CPU cost of NEC-4 increases geometrically with the line length. When the length is $1000 \mathrm{~m}$, about $1650 \mathrm{~s}$ is needed to calculate the results for a single frequency, which can become unaffordable when it is applied to solve a timedomain solution. At the same time, since the proposed method adopts a semi-analytical scheme in which numerical processes are only applied to two very short auxiliary lines, a much higher computational efficiency is obtained. It can be seen that only $3.2 \mathrm{~s}$ is needed when applying the proposed method in the case of a 1000-m long line.

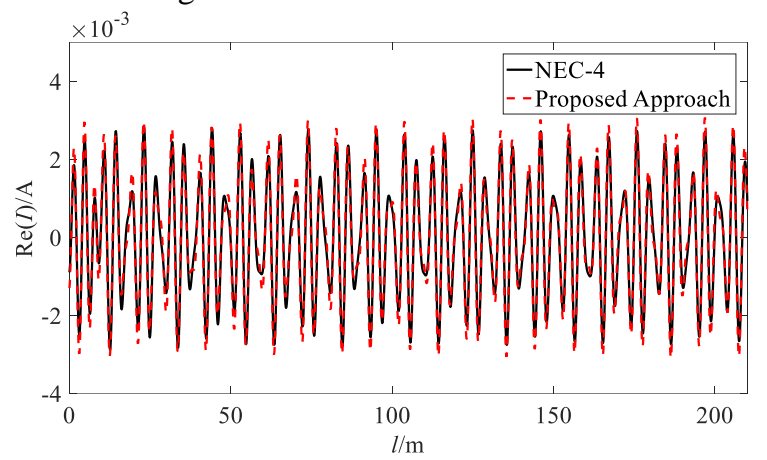

(a) $l=0 \sim 210 \mathrm{~m}$.

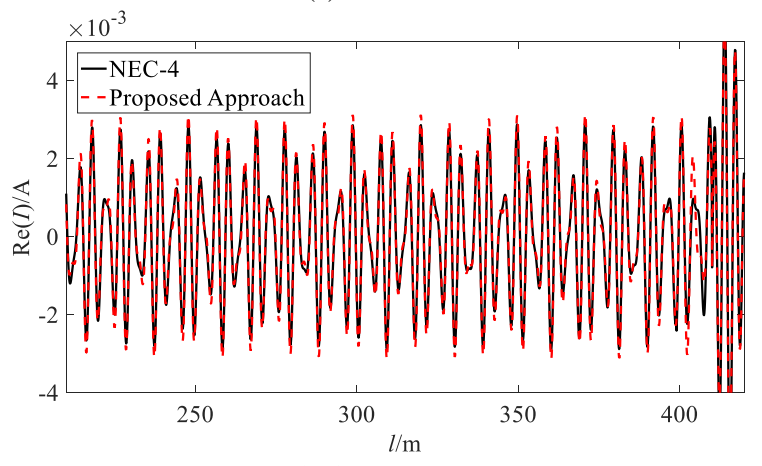

(b) $l=210 \sim 420 \mathrm{~m}$.

Fig. 8. Comparison between the real part solutions obtained using proposed approach and that using NEC-4. Same as Fig. 7 but with a line length of $400 \mathrm{~m}$.

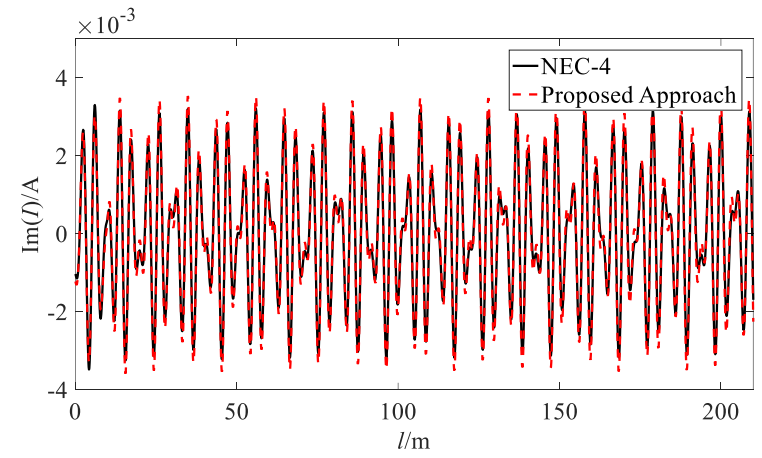

(a) $l=0 \sim 210 \mathrm{~m}$.

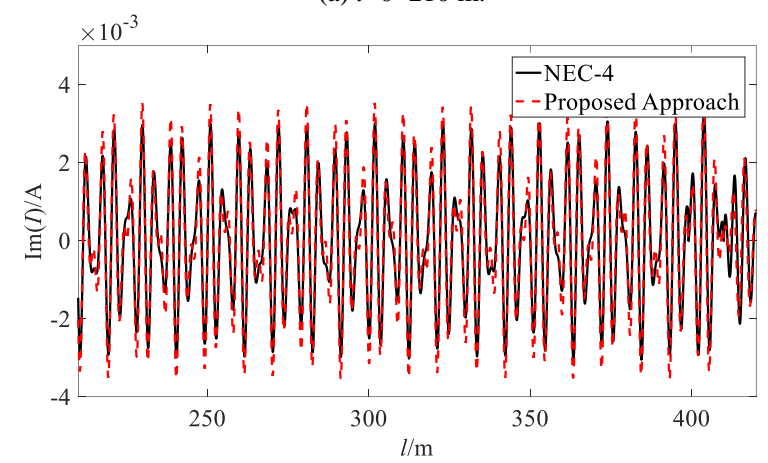

(b) $l=210 \sim 420 \mathrm{~m}$.

Fig. 9. Comparison between the imaginary part using proposed approach and that using NEC-4. Same as Fig. 7 but with a line length of $400 \mathrm{~m}$.

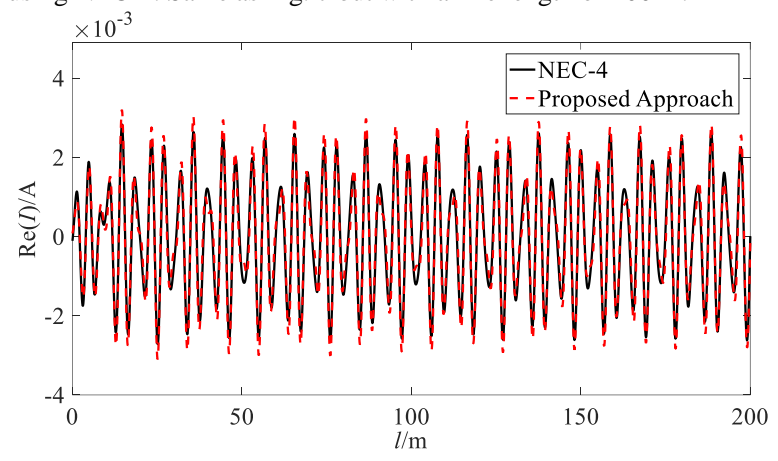

(a) $l=0 \sim 200 \mathrm{~m}$.

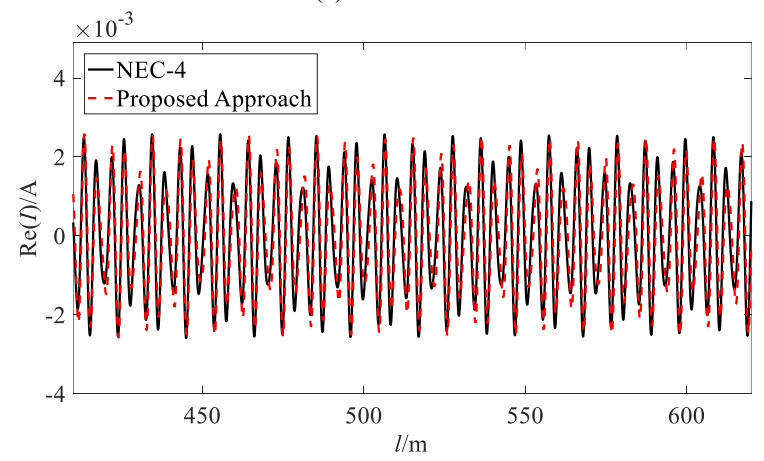

(b) $l=410 \sim 620 \mathrm{~m}$. 


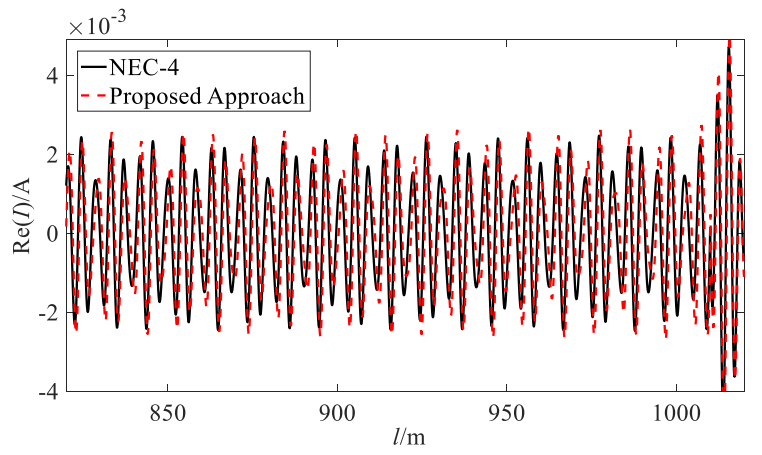

(c) $l=820 \sim 1020 \mathrm{~m}$.

Fig. 10. Comparison between the real part solutions obtained using proposed approach and that using NEC-4. Same as Fig. 7 but with a line length of 1000 m.

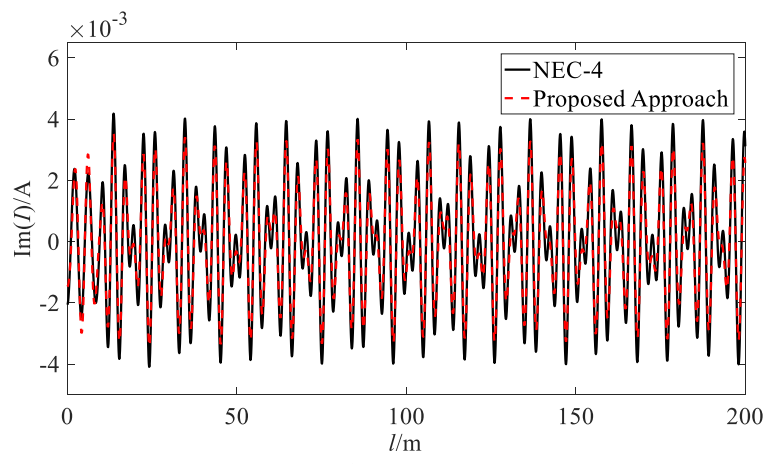

(a) $l=0 \sim 200 \mathrm{~m}$.

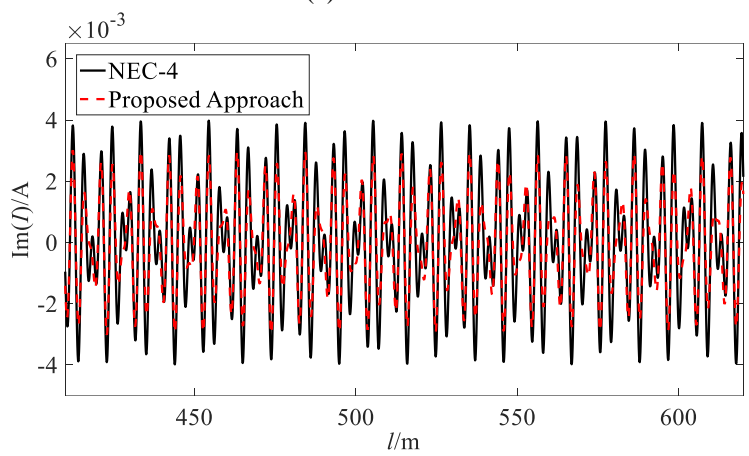

(b) $l=410 \sim 620 \mathrm{~m}$.

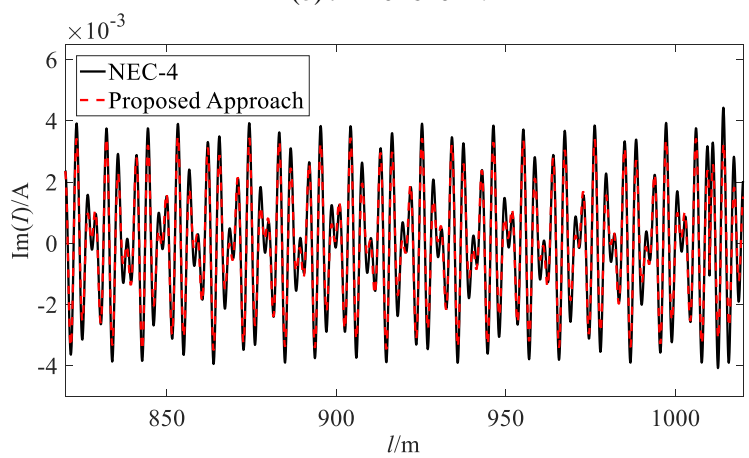

(c) $l=820 \sim 1020 \mathrm{~m}$.

Fig. 11. Comparison between the imaginary part solutions obtained using proposed approach and that using NEC-4. Same as Fig. 7 but with a line length of $1000 \mathrm{~m}$

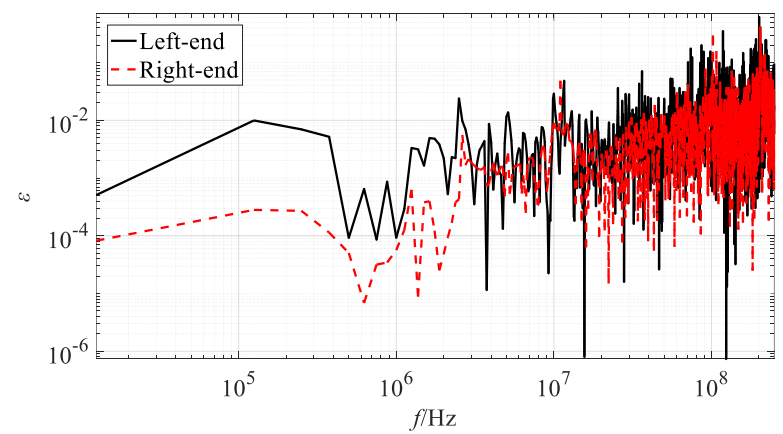

Fig. 12. Relationship between the relative error and the frequency with a line length of $1000 \mathrm{~m}$.

TABLE II

THE CPU Cost OF THE Two Methods (Proposed APPROACH VERSUS NEC4) AS A FUNCTION OF LINE LENGTH WITH FREQUENCY OF $100 \mathrm{MHZ}$ (UNIT: S)

\begin{tabular}{cccccc}
\hline & NEC-4 & \multicolumn{4}{c}{ Proposed Approach } \\
$200 \mathrm{~m}$ & $400 \mathrm{~m}$ & $1000 \mathrm{~m}$ & $200 \mathrm{~m}$ & $400 \mathrm{~m}$ & $1000 \mathrm{~m}$ \\
\hline 19.96 & 123.29 & 1650.43 & 3.17 & 3.18 & 3.20 \\
\hline
\end{tabular}

In order to investigate the solution in the time domain, the time-domain current solutions at the two terminal loads $Z_{1}$ and $Z_{2}$ are calculated. The IEC standard waveform is adopted as the waveform of the electric field of the incoming wave, which is defined in the IEC 61000-2-9 as

$$
E(t)=E_{0} k_{0}\left(e^{-\alpha t}-e^{-\beta t}\right)
$$

where $E_{0}=50 \mathrm{kV} / \mathrm{m}, k_{0}=1.05, \alpha=4 \times 10^{7} \mathrm{~s}^{-1}, \beta=6 \times 10^{8} \mathrm{~s}^{-1}$.

The full-wave approach (NEC-4), the classical TL method and the proposed method are adopted to calculate the current response at the two loads $Z_{1}$ and $Z_{2}$ in the frequency domain, and then the time domain solutions are obtained by using the IFFT method. The comparison among the time domain solutions for the induced current at the loads $Z_{1}$ and $Z_{2}$ is shown in Fig. 13. It can be seen that the results obtained using the proposed approach agrees very well with those obtained from NEC-4. On the other hand, the results calculated using the classical TL deviate significantly from the full-wave results. Moreover, it can be seen clearly that the classical TL method results in an underestimation of both of the gradient of the rising edge and the amplitude of the current response, in agreement with the findings of [14].

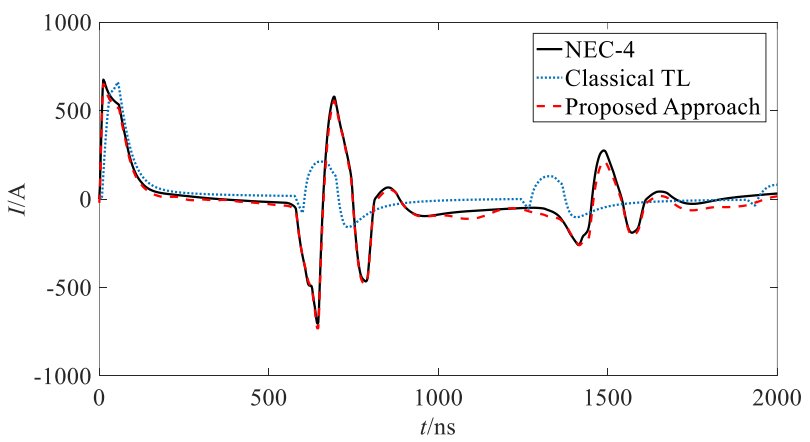

(a) 


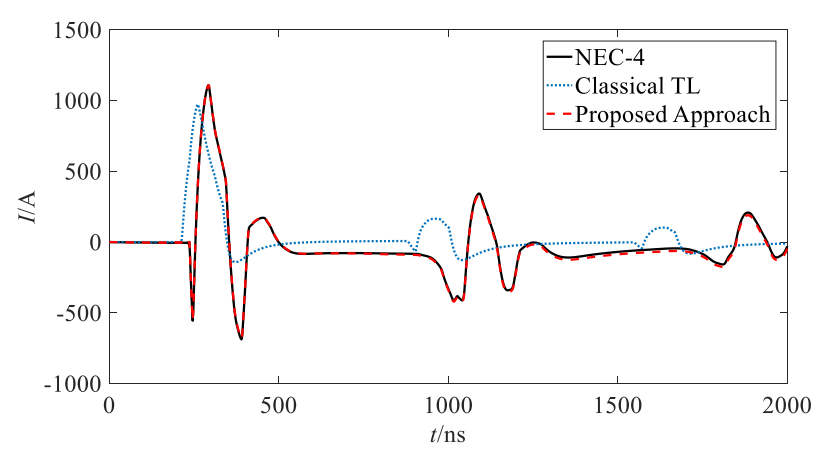

(b)

Fig. 13. Comparison between the proposed approach and results obtained using the classical TL approach and NEC-4. Induced current on a 1000-m long, 10$\mathrm{m}$ high, 1-mm diameter conductor above a ground of conductivity $10^{-2} \mathrm{~S} / \mathrm{m}$ and relative permittivity 10 . The line is excited by an IEC 61000-2-9 plane wave plane wave. The polarization angle, the azimuth angle and the elevation angle are $0^{\circ}, 0^{\circ}$ and $45^{\circ}$, respectively. (a) Induced current in the left-end impedance (b) Induced current in the right-end impedance.

\section{Example 3: Long Overhead Line above a Lossy Ground Excited by a Lumped Voltage Source}

We consider the case of a 100-m long, 10-m high, 1-mm diameter conductor above a lossy ground. The conductivity and the relative dielectric constant of the ground are assumed to be $0.01 \mathrm{~S} / \mathrm{m}$ and 10 , respectively. The terminal loads at both ends are $50 \Omega$. The line is excited by two $1-\mathrm{V}, 100 \mathrm{MHz}$ lumped voltage sources located at both terminal ends. The comparisons between the current solutions calculated using the proposed approach and those obtained using NEC-4 are shown in Fig. 14. It can be seen that the results calculated using the proposed method agrees very well with those obtained using NEC-4.

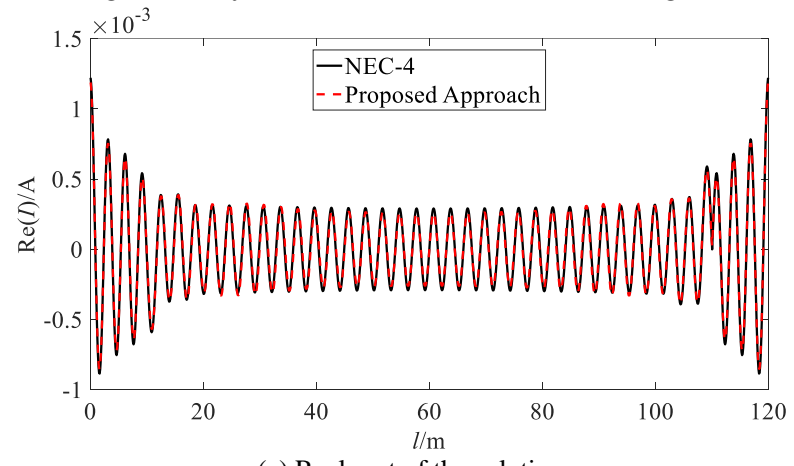

(a) Real part of the solution.

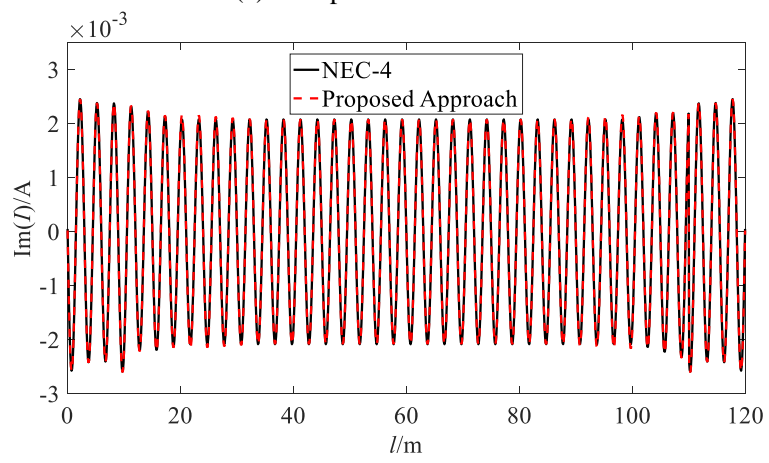

(b) Imaginary part of the solution.

Fig. 14. Comparison between the proposed approach and results obtained using NEC-4. Induced current on a 100-m long, 10-m high, 1-mm diameter conductor above a ground of conductivity $10^{-2} \mathrm{~S} / \mathrm{m}$ and relative permittivity 10 . The line is excited by a two $1-\mathrm{V}, 100-\mathrm{MHz}$ lumped voltage sources at each end of the line.

In the second case, the geometry of the line is the same like that in the first case, while only one lumped voltage source which equal to $1 \mathrm{~V}$ located at the right end of line. The comparison between the current solutions which calculated from the NEC-4 and proposed approach is shown in Figs. 15. It can be seen that the results calculated from the proposed method agrees well with that from the NEC-4.

\section{Example 4: Long Overhead Line above a Lossy Ground Terminated with a Nonlinear Load}

We consider the same line configuration of Section III.B. The terminal load at the left end is $50 \Omega$, while that at the right end is formed by the parallel connection of a $50 \Omega$ load with a nonlinear load. The $U-I$ characteristic of the nonlinear load is shown in Fig. 16 and corresponds to the behavior of $n$ typical voltage limiting equipment. The line is excited by the same IEC plane wave. According to the proposed method in Section II, the equivalent circuit source $i_{s c}$ is calculated by using the asymptotic method when setting the nonlinear load as zero. The equivalent admittance $y_{\text {in }}$ is calculated when the line is excited by a lumped voltage source located at the right terminal. The current response is calculated using the proposed method, as well as using the classical TL method. The calculated current across the nonlinear load and the $50 \Omega$ load at the right terminal are shown in Fig. 17.

It can be seen from the Fig. 17 that the classical TL approach results in an inaccurate and underestimated induced current. In particular, the amplitude of the current through the nonlinear load calculated using the proposed method is $890 \mathrm{~A}$ while that obtained using the classical TL theory is $720 \mathrm{~A}$.

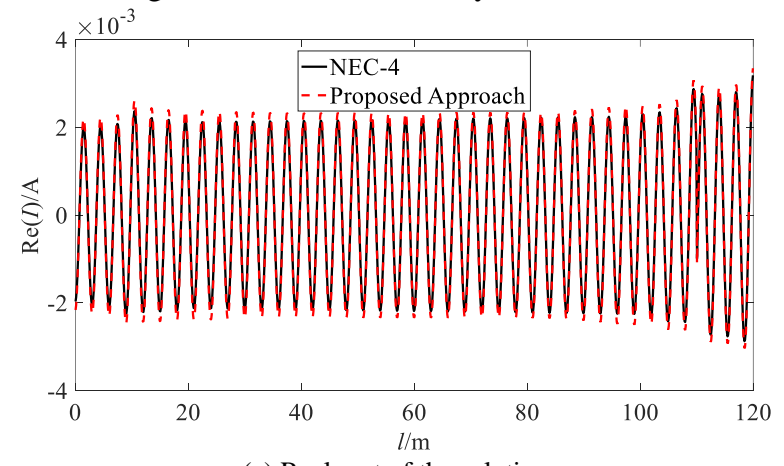

(a) Real part of the solution.

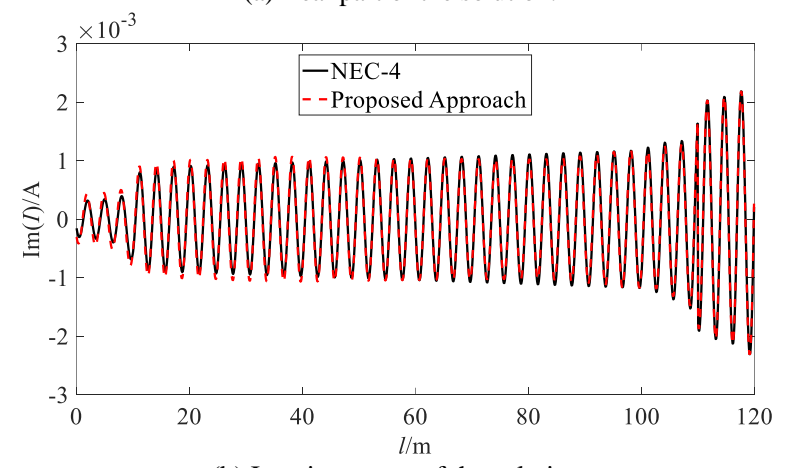

(b) Imaginary part of the solution.

Fig. 15. Same as in Fig. 14 but with only one voltage source located at the line right-end. 


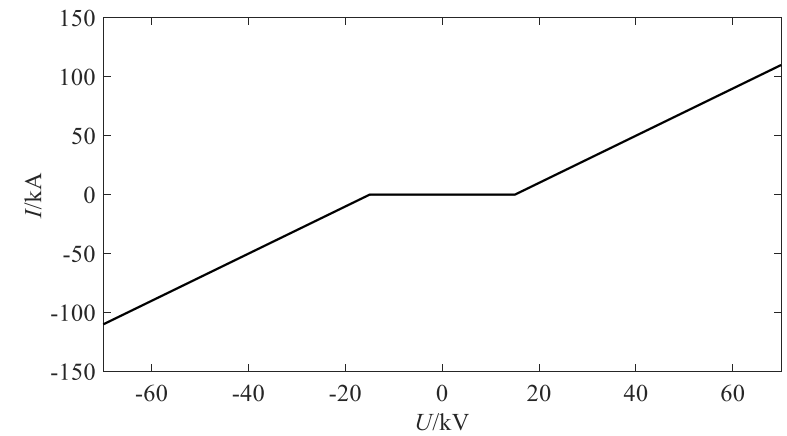

Fig. 16. $U-I$ characteristic of the nonlinear load.

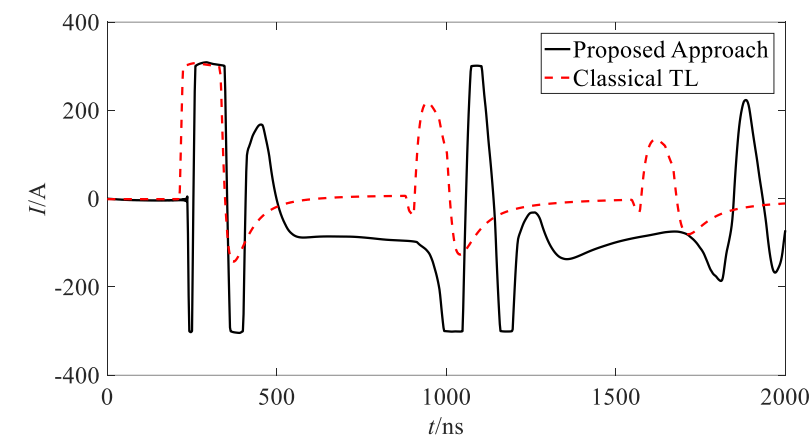

(a) Current through the $50 \Omega$ load.

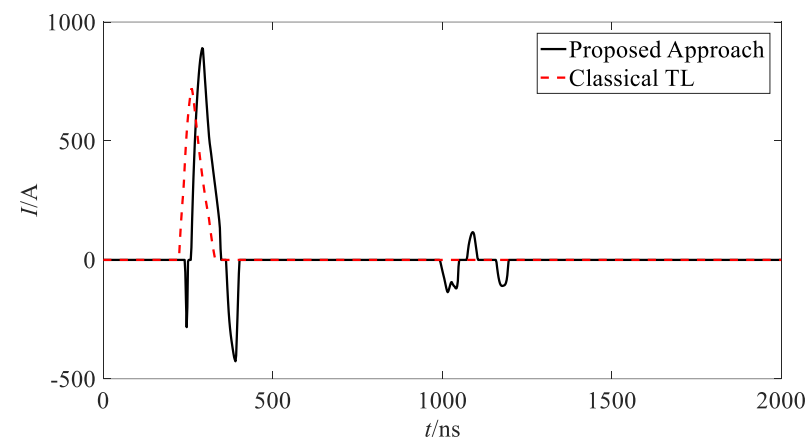

(b) Current through nonlinear load.

Fig. 17. Induced current in the right terminal (a) across the $50 \Omega$ load and (b) the nonlinear load. 100-m long, 10-m high, 1-mm diameter conductor above a ground of conductivity $10^{-2} \mathrm{~S} / \mathrm{m}$ and relative permittivity 10 . The line is excited by an IEC 61000-2-9 plane wave. The polarization angle, the azimuth angle and the elevation angle are $0^{\circ}, 0^{\circ}$ and $45^{\circ}$, respectively

\section{CONCLUSION}

This paper proposed an efficient method to model the high frequency electromagnetic field coupling to long overhead line terminated with nonlinear loads above a lossy ground. The cross-section of the line can be comparable to the wavelength of the incoming wave. In the proposed method, the asymptotic approach which is a semi-analytical method is adopted and extended to the case of a lossy ground. General solutions along the asymptotic region for the case of a lossy ground case are developed. The scattering and reflection coefficients of the line are fitted from the numerical results obtained by using a full wave solver to auxiliary lines with short line lengths. Once the coefficients are determined, the response of the entire line can be calculated analytically, resulting in a high computational efficiency compared to full wave solvers, especially for long lines. Since the asymptotic method is a frequency-domain approach, the time marching method which is a mixed frequency and time domain method is adopted to handle nonlinear loads.

The proposed method has been validated considering different numerical examples, and taking as reference full-wave simulations obtained by a full-wave solver based on the Method of Moments (NEC-4). The results showed that the proposed method can accurately and efficiently predict the response of a long lines to either lumped sources or external field excitation.

Future work will be devoted to investigate the numerical performance of the proposed method. The method will also be extended to the case of a multi-conductor transmission lines, inhomogeneous (multilayered) soil, and nonuniform excitation sources. Moreover, the use of more accurate expressions for the ground impedance instead of the Sunde's formula will also be investigated.

\section{REFERENCES}

[1] W. E. Scharfman, E. F. Vance, K. A. Graf, "EMP Coupling to Power Lines," IEEE Transaction on Antenna propagation, vol. 26, no. 1, pp. 129-135, Jan. 1978.

[2] F. M. Tesche, P. R. Barnes, "Transient Response of a Distribution Circuit Recloser and Control Unit to a high-Altitude Electromagnetic Pulseand Lightning," IEEE Transactions on Electromagnetic Compatibility, Vol. 32, No. 2, pp. 113-124, May 1990.

[3] C. A. Nucci, F. Rachidi, Interaction of electromagnetic fields with electrical networks generated by lightning, Chapter 12 of "The Lightning Flash ", 2nd Edition, IET, London, pp. 559-610, 2014.

[4] W. Radasky, "Fear of frying electromagnetic weapons threaten our data networks. Here's how to stop them," Spectrum, IEEE, vol. 51, pp. 46-51, 2014.

[5] F. Rachidi, "A Review of Field-to-Transmission Line Coupling Models with Special Emphasis to Lightning-Induced Voltages", IEEE Transactions on Electromagnetic Compatibility, Vol 54, No. 4, pp. 898 911, 2012.

[6] F. Rachidi, C. A. Nucci, M. Ianoz, and C. Mazzetti, "Influence of a lossy ground on lightning-induced voltages on overhead lines," IEEE Transactions on Electromagnetic Compatibility, vol. 38, no. 3, pp. 250264, Aug. 1996

[7] J. Guo, Y. Z. Xie, "An Efficient Model of Transient Electromagnetic Field Coupling to Multiconductor Transmission Lines Based on Analytical Iterative Technique in Time Domain", IEEE Transactions on Microwave Theory and Techniques, vol. 66, no. 66, pp. 2663 - 2673, 2018.

[8] A. Borghetti, F. Napolitano, C.A. Nucci, F. Rachidi, M. Rubinstein, Telegrapher's equations for field-to-transmission line interaction. Theory and application to the case of lightning induced voltages on distribution lines, Chapter 1 of "Advanced Techniques for Power System Modelling, Control and Stability Analysis", pp. 3-44, IET, London, 2016.

[9] C. R. Paul. Analysis of Multiconductor Transmission Lines, Second Edition, New York: John Wiley I\& Sons, 2008.

[10] F. M. Tesche, M. V. Ianoz, and T. Karlsson, EMC Analysis Methods and Computational Models. New York NY, USA: Wiley, 1997.

[11] C. D. Taylor, R. S. Satterwhite, C.W. Harrison, "The response of a terminated two-wire transmission line excited by a nonuniform electromagnetic field ", IEEE Transaction on Antenna propagation, Vol. AP-13, 1965.

[12] Agrawal A. K., H. J. Price, S. H. Gurbaxani, "Transient response of a multiconductor transmission line excited by a nonuniform electromagnetic field", IEEE Transactions on Electromagnetic Compatibility, Vol. EMC-22, No. 2, pp. 119-129, May 1980.

[13] F. Rachidi, "Formulation of the field-to-transmission line coupling equations in terms of magnetic excitation fields", IEEE Transactions on Electromagnetic Compatibility, Vol. 35, No. 3, Aug. 1993.

[14] D. Poljak, A. Shoory, F. Rachidi, S. Antonijevic, and S. Tkachenko, "Time domain generalized telegrapher's equations for the electromagnetic field coupling to finite length wires above a lossy 
ground," IEEE Transactions on Electromagnetic Compatibility, vol. 54, no. 1, pp. 218-224, Feb. 2012.

[15] T. J. Cui and W. C. Chew, "A full-wave model of wire structures with arbitrary cross sections," IEEE Transactions on Electromagnetic Compatibility, vol. 45, no. 4, pp. 626-635, Nov. 2003.

[16] S. Tkachenko, F. Rachidi, and M. Ianoz, "Electromagnetic field coupling to a line of finite length: Theory and fast iterative solutions in frequency and time domains," IEEE Transactions on Electromagnetic Compatibility, vol. 37, no. 4, pp. 509-518, Nov. 1995.

[17] S. Tkachenko, F. Rachidi, and M. Ianoz, "High-frequency electromagnetic field coupling to long terminated lines," IEEE Transactions on Electromagnetic Compatibility, vol. 43, no. 2, pp. 117129, May 2001.

[18] F. Middelstaedt, Sergey V. Tkachenko, R. Vick, "Transmission Line Reflection Coefficient Including High Frequency Effects," IEEE Transaction on Antenna propagation, 2018.

[19] J. Guo, Y.Z. Xie, A. C.i Qiu, "Calculation of Lightning Induced Voltages on Overhead Lines Using an Analytical Fitting Representation of Electric Fields", IEEE Transactions on Electromagnetic Compatibility, vol. 59, No. 3, pp. 879-886, Jun. 2017.

[20] J. Guo, Y. Z. Xie, F. Rachidi, K. J. Li, S. F. Wang, "On Nonuniform Transient Electromagnetic Field Coupling to Overhead Transmission Lines," IEEE Transaction on Antenna propagation, vol. 66, no. 6, pp. 3087-3096, Jun. 2018.

[21] J. Guo, Y. Z. Xie, F. Rachidi, "A Semi-Analytical Method to Evaluate Lightning-Induced Overvoltages on Overhead Lines Using the Matrix Pencil Method," IEEE Transactions on Power Delivery, vol. 33, no. 6, pp. 2837-2848, 2018.

[22] D. Poljak and C. Y. Tham, Integral Equation Techniques in Electromagnetics. Wessex, U.K.: WIT Press, 2003

[23] E. D. Sunde, Earth Conduction Effects in Transmission Systems. New York: Dover, 1968

[24] G. Bridges and L. Shafai, "Plane wave coupling to multiple conductor transmission lines above a lossy earth," IEEE Transactions on Electromagnetic Compatibility, vol. 31, no. 1, pp. 21-33, Feb. 1989.

[25] L. C. Shen, T. T. Wu, R. W. King, "A Simple Formula of Current in Dipole Antennas," IEEE Transaction on Antenna propagation, vol. 16, no. 5, pp. 542-547, Jan. 1968.

[26] R. G. Olsen, J. L. Young and D. C. Chang, "Electromagnetic Wave Propagation on a Thin Wire Above Earth," IEEE Transaction on Antenna propagation, vol. 28, no. 1, pp. 1413-1419, Sep. 2000.

[27] J. H. Richmond, "Green's Function Technique for Near-Zone Scattering by Cylindrical Wires with Finite Conductivity," IEEE Transaction on Antenna propagation, vol. 48, no. 9, pp. 114-117, Jan. 1980.

[28] J. R. Wait, "Theory of wave propagation along a thin wire parallel to an interface, Radio Science, vol. 7, no. 6, pp. 675-679, Jun. 1972.

[29] J. R. Wait, "Tutorial note on the general transmission line theory for a thin wire above the ground," IEEE Transactions on Electromagnetic Compatibility, vol. 33, pp.65-67, Feb. 1991.

[30] J. Rahman and T. K. Sarkar, "Deconvolution and total least squares in finding the impulse response of an electromagnetic system from measured data," IEEE Transaction on Antenna propagation, vol. 43, no. 4, pp. 416421, Apr. 1995.

[31] G. Lugrin, S. V. Tkachenko, F. Rachidi, M. Rubinstein, R. Cherkaoui, "High-Frequency Electromagnetic Coupling to Multiconductor Transmission Lines of Finite Length," IEEE Transactions on Electromagnetic Compatibility, Vol. 57, No. 6, pp. 1714-1723, Dec. 2015.

[32] J. Guo, Y. Z. Xie, A. C. Qiu, "JOR Iterative Method for the Modeling of MTLs Excited by EMP," IEEE Antennas and Wireless Propagation Letters, Vol. 15, pp. 536-539, Mar. 2016.

[33] J. Guo, Y. Z. Xie, F. G. Canavero, "Gauss-Seidel Iterative Solution of Electromagnetic Pulse Coupling to Three-Conductor Transmission Lines," IEEE Transactions on Electromagnetic Compatibility, Vol. 57, No. 2, pp. 292-298, Dec. 2015.

[34] F. Rachidi and S. Tkachenko, Electromagnetic Field Interaction With Transmission Lines: From Classical Theory to HF Radiation Effects. Boston, MA, USA: WIT Press, 2008.

[35] S. Tkachenko, J. B. Nitsch, R. Rambousky, "Use of the TransmissionLine Super Theory to Determine Generalized Reflection and Transmission Coefficients of an Inhomogeneous Line with Risers", Interaction Notes, Note 626, March 2015. (http://eceresearch.unm.edu/summa/notes/In/IN626.pdf )

[36] J. B. Nitsch, R. Rambousky, S. Tkachenko, "Introduction of Reflection and Transmission Coefficients for Nonuniform Radiating Transmission
Lines", IEEE Transactions on Electromagnetic Compatibility, vol. 57, no. 6, pp. 1705-1712, Dec. 2015.

[37] T. K. Sarkar, D. D. Weiner, "Scattering Analysis of Nonlinearly Loaded Antennas," IEEE Transaction on Antenna propagation, vol. 41, no. 1, pp. 125-131, Jan. 1976

[38] T. K. Liu, F. M. Tesche, "Analysis of Antennas and Scatterers with Nonlinear Loads," IEEE Transaction on Antenna propagation, vol. 41, no. 1, pp. 131-139, Jan. 1976.

[39] Burke J. Numerical Electromagnetics Code-NEC-4, Method of Moments, Part I: User's Manual and Part II: Program Description-Theory. Lawrence Livermore National Laboratory, Livermore, California, USA, 1992.

[40] H. Y. Xue, A. Ametani, J. Mahseredjian, Y. Baba, F. Rachidi, I. Kocar, "Transient Responses of Overhead Cables Due to Mode Transition in High Frequencies," IEEE Transactions on Electromagnetic Compatibility, Vol. 60, No. 3, pp. 785-794, Jun. 2018.

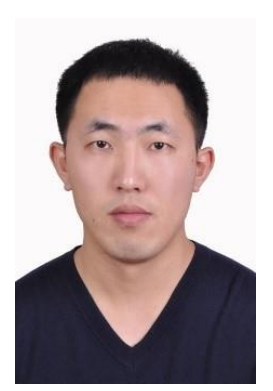

Jun Guo (S'12-M'16) was born in Shanxi, China, in 1986. He received the $\mathrm{Ph} . \mathrm{D}$. degree in electrical engineering from Xi'an Jiaotong University, Shannxi, China, in July 2016. From 2017 to 2018, he was a postdoctor in the EMC Laboratory, Swiss Federal Institute of Technology, Lausanne, Switzerland. He is currently a Lecturer of School of Electrical Engineering, Xi'an Jiaotong University, China. His research interests include transient analysis of multiconductor transmission lines and transient electromagnetic field measurement, etc. He has received several awards including the IEEE Outstanding Young Scientist Award from the 2018 Joint IEEE EMC \& APEMC Symposium, and the URSI Young Scientist Award from the 2019 URSI AP-RASC, etc.

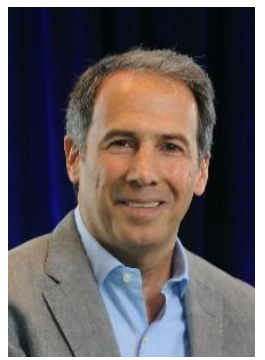

Farhad Rachidi (M'93-SM'02-F'10) received the M.S. degree in electrical engineering and the Ph.D. degree from the Swiss Federal Institute of Technology, Lausanne, Switzerland, in 1986 and 1991, respectively. He was with the Power Systems Laboratory, Swiss Federal Institute of Technology, until 1996. In 1997, he joined the Lightning Research Laboratory, University of Toronto, Toronto, ON, Canada. From 1998 to 1999, he was with Montena EMC, Rossens, Switzerland. He is currently a Titular Professor and the Head of the EMC Laboratory with the Swiss Federal Institute of Technology, Lausanne, Switzerland. He has authored or coauthored over 160 scientific papers published in peer-reviewed journals and over 350 papers presented at international conferences.

Dr. Rachidi is currently a member of the Advisory Board of the IEEE TRANSACTIONS ON ELECTROMAGNETIC COMPATIBILITY and the President of the Swiss National Committee of the International Union of Radio Science. He has received numerous awards including the 2005 IEEE EMC Technical Achievement Award, the 2005 CIGRE Technical Committee Award, the 2006 Blondel Medal from the French Association of Electrical Engineering, Electronics, Information Technology and Communication (SEE), the 2016 Berger Award from the International Conference on Lightning Protection, the 2016 Best Paper Award of the IEEE Transactions on EMC, and the 2017 Motohisa Kanda Award for the most cited paper of the IEEE Transactions on EMC. In 2014, he was conferred the title of Honorary Professor of the Xi'an Jiaotong University in China. He served as the Vice-Chair of the European COST Action on the Physics of Lightning Flash and its Effects from 2005 to 2009, the Chairman of the 2008 European Electromagnetics International Symposium, the President of the International Conference on Lightning Protection from 2008 to 2014, the Editor-in-Chief of the Open Atmospheric Science Journal (2010-2012) and the Editor-in-Chief of the IEEE TRANSACTIONS ON ELECTROMAGNETIC COMPATIBILITY from 2013 to 2015 . 


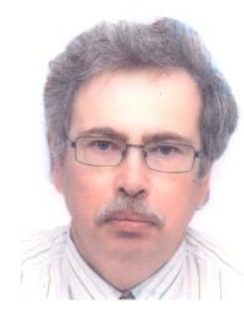

Sergey V. Tkachenko (M'07-SM'12) was born in Moscow, Russia, in 1958. He received the M.S. and $\mathrm{Ph} . \mathrm{D}$. degrees in mathematical and theoretical physics from the Moscow Engineering and Physics Institute, Moscow, Russia, in 1981 and 1987, respectively. From 1986 to 1989, he was a Researcher and a Senior Researcher with the Department of Electromagnetic Compatibility, State Radio Research Institute, Moscow. In 1993, he was an Invited Researcher at the Swiss Federal Institute of Technology (EPFL), Lausanne, Switzerland, for eight months. From 1996 to 1999, he worked with the LRE EPFL within a framework of the European INTAS Project 94-3939 "Electromagnetic Compatibility of Interconnection Cables Subject to Interferences." Since 2000, he has been an Invited Researcher with the Institute for Fundamental Electrical Engineering and EMC (Chair of Electromagnetic Compatibility now), Otto-von-Guericke University, Magdeburg, Germany. He is an author or coauthor of more than 130 scientific papers published in peerreviewed journals and presented at international conferences. His research interests include applied electromagnetics and electromagnetic compatibility, in particular electromagnetic field coupling to complex wiring structures, also inside cavities or (and) with stochastic geometry.

Dr. Tkachenko is the coeditor (together with F. Rachidi) of the book "Electromagnetic Field Interaction with Transmission Lines: From Classical Theory to HF Radiation Effects" (Southampton, U.K.: WIT Press, 2008). He, together with J. Nitsch, has received the Best HPE Paper Award three times (best basic paper for the years 2002-2003, 2006-2007, and 2008-2009, correspondingly). He is an elected EMP Fellow (2008, for theoretical investigation of high-frequency electromagnetic field coupling to transmission lines in different environments)

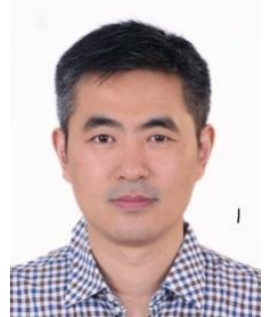

Yan-zhao Xie (M'12) was born in Henan, China, in 1973. He received the Ph.D. degree in electrical engineering from Tsinghua University, Beijing, China, in December, 2005. He is currently a Professor of School of Electrical Engineering, Xi'an Jiaotong University, China. His research interests include electromagnetic compatibility, electromagnetic transients in power system and high-power electromagnetics, etc. He has been the director of National Center for International Research on Transient Electromagnetics and Applications (TEA) since 2016. 